\title{
Long-Distance Entanglement of Spin Qubits via Ferromagnet
}

\author{
Luka Trifunovic, Fabio L. Pedrocchi, and Daniel Loss \\ Department of Physics, University of Basel, Klingelbergstrasse 82, CH-4056 Basel, Switzerland \\ (Received 18 February 2013; revised manuscript received 4 August 2013; published 4 December 2013)
}

\begin{abstract}
We propose a mechanism of coherent coupling between distant spin qubits interacting dipolarly with a ferromagnet. We derive an effective two-spin interaction Hamiltonian and find a regime where the dynamics is coherent. Finally, we present a sequence for the implementation of the entangling controlledNOT gate and estimate the corresponding operation time to be a few tens of nanoseconds. A particularly promising application of our proposal is to atomistic spin qubits such as silicon-based qubits and nitrogenvacancy centers in diamond to which existing coupling schemes do not apply.
\end{abstract}

DOI: 10.1103/PhysRevX.3.041023

\section{INTRODUCTION}

Quantum coherence and entanglement lie at the heart of quantum-information processing. One of the basic requirements for implementing quantum computing is to generate, control, and measure entanglement in a given quantum system. This implementation is a rather challenging task, as it requires us to overcome several obstacles, the most important one being decoherence processes. These negative effects have their origin in the unavoidable coupling of the quantum systems to the environment in which they reside.

A guiding principle in the search for a good system to encode qubits is the smaller the system, the more coherence - or, more precisely, the fewer degrees of freedom, the weaker the coupling to the environment. Simultaneously, one needs to be able to coherently manipulate the individual quantum objects, which is more efficient for larger systems. These opposing requirements immediately force us to compromise between manipulation and decoherence requirements.

Following this principle, among the most promising candidates for encoding a qubit, we find atomistic twolevel systems, such as nitrogen-vacancy (NV) centers and silicon-based spin qubits [1-12]. The latter are composed of nuclear (electron) spins of phosphorus atoms in a silicon nanostructure. They have very long $T_{2}$ times of $60 \mathrm{~ms}$ [13] for nuclei and of $200 \mu$ s for electrons [14]. Recently, highfidelity single-qubit gates and readout have been demonstrated experimentally [14]. Nitrogen-vacancy centers [15] in diamond have also been demonstrated experimentally to be very stable, with long decoherence times of $T_{2}^{*} \approx 20 \mu \mathrm{s}$ and $T_{2} \approx 1.8 \mathrm{~ms}$ [16]. Both types of spin qubit have the additional advantage that noise due to surrounding nuclear spins can be avoided by isotopically purifying the material.

Published by the American Physical Society under the terms of the Creative Commons Attribution 3.0 License. Further distribution of this work must maintain attribution to the author(s) and the published article's title, journal citation, and DOI.
Subject Areas: Magnetism, Quantum Information

Unfortunately, it is difficult to make these spin qubits interact with each other in a controlled and scalable fashion $[17,18]$. They are very localized, and their position in the host material is given and cannot be adjusted easily. Therefore, if, during their production, two qubits turn out to lie close to each other, they will always be coupled, while if they are well isolated from each other, they will never interact. It is thus of high interest to propose a scheme to couple such atomistic qubits in a way that allows a high degree of control.

We fill this gap in the present work by proposing a setup to couple two spin qubits separated by a relatively large distance on the order of micrometers; see Fig. 1. The coupling is mediated via a ferromagnet with gapped excitations to which the spin qubits are coupled by magnetic dipole-dipole interaction. Since the ferromagnet is gapped, only virtual magnons are excited, but in order to obtain a sizable coupling, one needs to tune the splitting of the qubit close to ferromagnetic resonance (FMR). Another possibility could be to use magnonic crystals in place of the ferromagnet [19]. The on-and-off switching of the qubit-qubit interaction is therefore achieved by tuning qubits on and off resonance (see below). The resulting system is thus

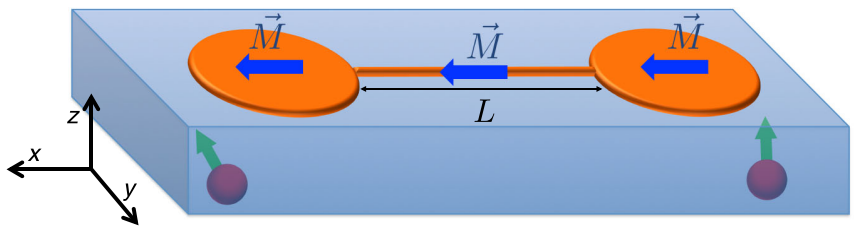

FIG. 1. The schematics of the ferromagnetic coupler setup. The orange dogbone shape denotes the ferromagnet that is coupled via magnetic dipole interaction to spins of nearby quantum dots (red spheres with green arrows). The ferromagnet is assumed to be a monodomain, and its magnetization is denoted by blue arrows $(M)$ that can take arbitrary orientation. $L$ is the length of the quasi-1D ferromagnetic channel that is approximately equal to the distance between the qubits. The shape of the ferromagnetic coupler is chosen such that it enables strong coupling to the spin qubits while maintaining the spatially slowly decaying 1D susceptibility between the two disks. 
realizable with present state-of-the-art technologies. We point out that our analysis is not restricted to a precise type of spin qubit but is, in principle, applicable to any system that dipolarly interacts with the spins of a ferromagnet. In particular, our proposal is also applicable to an electron spin localized in a semiconductor quantum dot or in a double quantum dot [20], gate defined or self-assembled $[21,22]$. While other schemes exist to couple such qubits over large distances [23-27], none of them is applicable to atomistic qubits. The main novelty of our proposal is thus the possibility to also couple atomistic qubits that are of high technological relevance.

Before we proceed with the quantitative analysis, let us first give an intuitive picture of the qubit-qubit coupling. The coupling between two distant qubits is mediated via a coupler system. The relevant quantity of this coupler is its spin-spin susceptibility-in order to have a long-range coupling, a slowly spatially decaying susceptibility is required. The dimensionality of the coupler plays an important role since, in general, it strongly influences the spatial decay of the susceptibility-this behavior can be anticipated from purely geometric considerations. Furthermore, since the coupler interacts with the qubits via magnetic dipolar forces, we require that a large part of the coupler lie close to the qubits. To this end, we immediately see that the dogbone shape depicted in Fig. 1 satisfies these two requirements-strong dipolar coupling to the qubits while retaining a slow, practically $1 \mathrm{D}$, spatial decay of the susceptibility at separation between the qubits.

\section{MODEL}

The system we consider consists of two spin- $\frac{1}{2}$ qubits coupled dipolarly to the ferromagnet, with the following Hamiltonian:

$$
H=H_{\sigma}+H_{F}+H_{I},
$$

where $H_{F}$ is the for now unspecified Hamiltonian of the dogbone-shaped ferromagnet that is assumed to be polarized along the $x$ axis. We first assume that the qubits are also polarized along the $x$ axis $H_{\sigma}=\sum_{i=1,2} \frac{\Delta_{i}}{2} \sigma_{i}^{x}$, while the ferromagnet disk axes are along $z$; see Fig. 1 . The magnetic dipole coupling between the ferromagnet and the spin qubits can be written as

$$
\begin{aligned}
H_{I}= & \frac{\mu_{B} M_{s}}{4 \pi S} \sum_{i=1,2} \int d \mathbf{r} S_{\mathbf{r}}^{x}\left[\left(\frac{3 i A_{i, \mathbf{r}}^{\prime}}{2}+\frac{3 C_{i, \mathbf{r}}^{\prime \prime}}{4}\right) \sigma_{i}^{+}+\right.\text {H.c. } \\
& \left.+\frac{1}{2}\left(B_{i, \mathbf{r}}-3 C_{i, \mathbf{r}}^{\prime}\right) \sigma_{i}^{x}\right]+S_{\mathbf{r}}^{+}\left[\left(\frac{3}{8} C_{i, \mathbf{r}}^{\prime}-\frac{3 i}{2} A_{i, \mathbf{r}}^{\prime \prime}+\frac{3}{8} B_{i, \mathbf{r}}\right) \sigma_{i}^{+}\right. \\
& \left.-\frac{1}{8}\left(B_{i, \mathbf{r}}-3 C_{i, \mathbf{r}}^{\prime}\right) \sigma_{i}^{-}+\left(\frac{3 C_{i, \mathbf{r}}^{\prime \prime}}{4}+\frac{3 i A_{i, \mathbf{r}}^{\prime}}{2}\right) \sigma_{i}^{x}\right]+ \text { H.c. },
\end{aligned}
$$

where $\mu_{B}$ is the Bohr magneton, $M_{s}$ is the saturation magnetization of the ferromagnet, and $A_{\mathbf{r}}, B_{\mathbf{r}}$, and $C_{\mathbf{r}}$ are given by

$$
\begin{gathered}
A_{\mathbf{r}}=\frac{r^{z} r^{+}}{r^{5}}, \\
C_{\mathbf{r}}=\frac{\left(r^{+}\right)^{2}}{r^{5}}, \\
B_{\mathbf{r}}=\frac{1}{r^{3}}\left(2-\frac{3 r^{+} r^{-}}{r^{2}}\right),
\end{gathered}
$$

with $S_{\mathbf{r}}^{ \pm}=S_{\mathbf{r}}^{y} \pm i S_{\mathbf{r}}^{z}, \sigma^{ \pm}=\sigma^{y} \pm i \sigma^{z}$. Here, we denote the real part of a complex number with a prime and the imaginary part with a double prime. The operator $\boldsymbol{S}_{\mathbf{r}}$ describes the spin of the ferromagnet at the position $\mathbf{r}$.

Next, we release the assumptions about the mutual orientation of the disk axes, the axes of polarization of the ferromagnet, and the direction of the qubit splitting and assume that these axes can take arbitrary directions. Now, the interaction Hamiltonian reads

$$
\begin{aligned}
H_{I}= & \frac{\mu_{B} M_{s}}{S} \sum_{i=1,2} \int d \mathbf{r} S_{\mathbf{r}}^{\tilde{z}}\left[a_{i, \mathbf{r}} \sigma_{i}^{z}+b_{i, \mathbf{r}} \sigma_{i}^{+}+\text {H.c. }\right] \\
& +S_{\mathbf{r}}^{\tilde{+}}\left[c_{i, \mathbf{r}} \sigma_{i}^{z}+d_{i, \mathbf{r}} \sigma_{i}^{+}+e_{i, \mathbf{r}} \sigma_{i}^{-}\right]+\text {H.c., }
\end{aligned}
$$

where $S_{\mathbf{r}}$ has a quantization axis along $\tilde{z}$ and $\sigma^{ \pm}=\sigma^{x} \pm$ $i \sigma^{y}$. The coordinate systems $(x, y, z)$ for the qubit $\boldsymbol{\sigma}$ and $(\tilde{x}, \tilde{y}, \tilde{z})$ for the ferromagnet can be different. The expressions of the coefficients in Eq. (6) are now more complicated; nevertheless, it is important to note that the integrals of these coefficients are experimentally accessible. The qubits can be used to measure the stray field of the ferromagnet, which is given by $\boldsymbol{B}_{s}=-M_{s}\left(2 b_{i}^{\prime},-2 b_{i}^{\prime \prime}, a_{i}\right)$, where $\left\{a_{i}, \ldots, e_{i}\right\}=\int d \mathbf{r}\left\{a_{i}, \ldots, e_{i}\right\}_{\mathbf{r}}$. In order to measure the remaining coefficients, one needs to apply the magnetic field externally in order to sequentially polarize the ferromagnet along the two perpendicular directions to the ferromagnet easy axis. The coefficients are then obtained by measuring again the stray fields (with the aid of the qubits) that are now given by $-M_{s}\left(2 d_{i}^{\prime}+2 e_{i}^{\prime}, 2 e_{i}^{\prime \prime}-2 d_{i}^{\prime \prime}, 2 c_{i}^{\prime}\right)$ and $-M_{s}\left(-2 d_{i}^{\prime \prime}-2 e_{i}^{\prime \prime}, 2 e_{i}^{\prime}-2 d_{i}^{\prime},-2 c_{i}^{\prime \prime}\right)$. Furthermore, all the results that we are going to obtain for the qubit-qubit coupling as well as for the decoherence time will depend only on the integrals of the coefficients, i.e., on $\left\{a_{i}, \ldots, e_{i}\right\}$ rather than on $\left\{a_{i}, \ldots, e_{i}\right\}_{\mathbf{r}}$. We point out that the stray field $\boldsymbol{B}_{s}$ induces a splitting on the qubits that is incorporated into $H_{\sigma}$.

\section{A. Coherent coupling}

We proceed to derive the effective qubit-qubit coupling by performing a Schrieffer-Wolff (SW) transformation [28]. We assume that the excitations in the ferromagnet are gapped 
because of some magnetic anisotropy (e.g., shape anisotropy) or externally applied magnetic field, with the gap being denoted by $\Delta_{F}$. (For FMR dependence on an externally applied magnetic field, see Ref. [29].) The presence of a gap is important because when the qubit splitting $\Delta$ is below the FMR frequency, flipping the qubit spin cannot excite magnons in the ferromagnet; thus, there are only virtual magnons excited via coupling to the qubits-otherwise, such a coupling would lead to strong decoherence in the qubits. Because of the presence of the gap in the ferromagnet, its transversal susceptibility $\chi_{\perp}(\omega, \mathbf{r})$ decays exponentially below FMR $\left(\omega<\Delta_{F}\right)$ with the characteristic magnetic length $l_{F} \propto 1 / \sqrt{\Delta_{F}-\omega}$; thus, we only take into account terms with $\omega \sim \Delta_{F}$-see Eqs. (B22) and (B24) in Appendix B. Straightforward application of lowest-order SW transformation accompanied by tracing out the degrees of freedom of the ferromagnet yields the effective qubit-qubitcoupling Hamiltonian

$$
\begin{aligned}
H_{\text {eff }}= & H_{\sigma}+\frac{\mu_{B}^{2} M_{s}^{2}}{S^{2}} \chi_{\perp}^{1 \mathrm{D}}\left(\Delta_{1}, L\right) e_{1} \sigma_{1}^{-}\left(c_{2} \sigma_{2}^{z}+d_{2} \sigma_{2}^{+}+e_{2} \sigma_{2}^{-}\right)^{\dagger} \\
& +1 \leftrightarrow 2+\text { H.c., }
\end{aligned}
$$

where $\chi_{\perp}^{1 \mathrm{D}}$ is the transverse susceptibility (i.e., transverse to the $\tilde{z}$ direction) of a quasi-1D ferromagnet, since we assume a dogbone-shaped ferromagnet. We neglect the longitudinal susceptibility $\chi_{\|}$since it is suppressed by temperature. We note that $\frac{\mu_{B}^{2} M_{s}^{2}}{S^{2}} \chi_{\perp}^{1 \mathrm{D}} \sim \frac{\mu_{B}^{2} M_{s}^{2}}{T_{c}} l_{F} e^{-L / l_{F}}$; thus, ferromagnets with high saturation magnetization and moderate Curie temperature are desirable for the strong coupling. Furthermore, it can be seen from the above expression that in order to obtain a sizable coupling between the qubits, we have to tune both the qubits close to resonance $\Delta_{i} \sim \Delta_{F}$ (see Sec. II B). This tuning can be achieved by applying an external magnetic field. Since the $g$ factor of the ferromagnet is not generally the same as the $g$ factor of the qubit, it is possible to tune the system on resonance with an external homogeneous magnetic field. The fine-tuning can then be achieved by locally applying a small external magnetic field from a coil. The on-resonance requirement offers an elegant way to switch on and off the coupling between the qubits. The idea is to tune the qubit splitting close to resonance for switching on the mediated interaction and to tune it off resonance to switch off the mediated interaction.

The expression for the transverse susceptibility of a ferromagnet is given in Eq. (B24) of Appendix B, assuming $H_{F}$ to be of Heisenberg type. The derivation given therein relies on the fact that the dispersion of low-lying ferromagnetic excitations (i.e., spin waves) is quadratic [30]. Note that for very long wavelengths (bigger than a micron), the excitations are so-called dipolar spin waves with dispersions very different from the quadratic one and dependent on concrete boundary conditions [31]. Since we are considering ferromagnets with dimensions not exceeding microns, the relevant excitations are exchangespin waves with quadratic dispersion that is practically independent of specific boundary conditions [31]. We note here that the FMR can increase toward the edges of the sample [32]; thus, one should use a ferromagnetic coupler with lateral dimensions bigger than the one quoted in Sec. IV in order to have a middle region with nearly spatially independent FMR.

Special care has to be taken for the validity of the perturbation theory employed herein, since we are working close to FMR; i.e., $\Delta-\Delta_{F}$ has to be small but still much larger than the coupling of a qubit to an individual spin of the ferromagnet. For the perturbation theory to be valid, we require the tilt of each ferromagnet spin to be sufficiently small (i.e., $\left\langle S_{\mathbf{r}}^{ \pm}\right\rangle \ll 1$ ). The tilt of the central spin of the ferromagnetic disk can be estimated by the integral over the dogbone disk $D$

$$
\left\langle S_{\mathbf{r}}^{ \pm}\right\rangle=\frac{\mu_{B}}{a^{3}} \int_{D} \chi_{\perp}(\mathbf{r}) B_{\perp}(\mathbf{r}) d \mathbf{r}
$$

where $a$ is the lattice constant of the ferromagnet and $B_{\perp}(\mathbf{r})$ is the perpendicular (to the magnetization direction) component of the field produced at position $\mathbf{r}$ by the qubit. Using cylindrical coordinates, we then obtain

$$
\left\langle S_{\mathbf{r}}^{ \pm}\right\rangle \sim \frac{\mu_{0} \mu_{B}^{2}}{a} \int_{0}^{R} \rho d \rho \frac{1}{\left(\rho^{2}+h^{2}\right)^{3 / 2}} \frac{S}{D \rho},
$$

where $\frac{S}{D \rho}$ is the spatial decay of the transversal susceptibility and $\frac{1}{\left(\rho^{2}+h^{2}\right)^{3 / 2}}$ is the decay of the dipolar field that causes the perturbation of the ferromagnet. For our choice of parameters, we obtain a tilt $\left\langle S_{\mathbf{r}}^{ \pm}\right\rangle<10^{-7} \ll 1$. Even though each spin is just slightly tilted, we obtain a sizable coupling due to a big number of spins involved in mediating the coupling.

For the sake of completeness, in Appendix D, we present a detailed discussion of the effective coupling mediated by the ferromagnet when the qubits are exchange coupled to the ferromagnet, which requires a tunnel coupling between spin qubit and ferromagnet.

\section{B. Implementation of two-qubit gates}

Two qubits interacting via the ferromagnet evolve according to the Hamiltonian $H_{\text {eff }}$; see Eq. (7). The Hamiltonian is therefore the sum of Zeeman terms and qubit-qubit interaction. These terms, by and large, do not commute, making it difficult to use the evolution to implement standard entangling gates. In order to obtain sizable coupling, we need to assume that the two qubits are on resonance with each other $\Delta_{1} \simeq \Delta_{2}$. Now, $H_{\sigma}$ acts only in the subspace spanned by $\{|\uparrow \uparrow\rangle,|\downarrow\rangle\rangle$ and the Zeeman splitting of the qubits is much larger than the effective qubitqubit coupling; we can thus neglect the effect of $H_{\text {eff }}$ in this part of the subspace and approximate it by its projection in the space spanned by the vectors $\{|\uparrow \downarrow\rangle,|\downarrow \uparrow\rangle\}$

$$
H_{\text {eff }}^{\prime}=H_{\sigma}+\alpha\left(\sigma_{1}^{x} \sigma_{2}^{x}-\sigma_{1}^{y} \sigma_{2}^{y}\right)+\kappa\left(\sigma_{1}^{x} \sigma_{2}^{y}+\sigma_{1}^{y} \sigma_{2}^{x}\right),
$$


where $\alpha=-8 \operatorname{Re}\left(e_{1} e_{2}^{*}\right)$ and $\kappa=-4 \operatorname{Re}\left(d_{1} e_{2}^{*}+d_{2} e_{1}^{*}\right)$. Within this approximation, the coupling in $H_{\text {eff }}^{\prime}$ and the Zeeman terms now commute. From here, we readily see that the stray field components $a_{i}$ and $b_{i}$, as well as the coefficient $c_{i}$, do not determine the operation time of the two-qubit gates- the operation time depends only on $d_{i}$ and $e_{i}$. To proceed, we perform a rotation on the second qubit around the $z$ axis by an angle $\tan \theta=\kappa / \alpha$ and arrive at the Hamiltonian

$$
H_{\mathrm{eff}}^{\prime}=H_{\sigma}+\sqrt{\alpha^{2}+\kappa^{2}}\left(\sigma_{1}^{x} \tilde{\sigma}_{2}^{x}-\sigma_{1}^{y} \tilde{\sigma}_{2}^{y}\right) .
$$

We consider the implementation of the isWAP gate $U_{\text {isWAP }}=e^{i\left(\sigma_{1}^{x} \tilde{\sigma}_{2}^{x}+\sigma_{1}^{y} \tilde{\sigma}_{2}^{y}\right) \pi / 4}$, which can be used to implement the controlled-NOT (CNOT) gate [33]. The Hamiltonian $H_{\text {eff }}^{\prime}$ can be transformed to the desired form by changing the sign of the $\sigma_{1}^{x} \tilde{\sigma}_{2}^{x}$ term. This change is achieved with the following sequence [34]:

$$
U_{i \mathrm{SWAP}}=\sigma_{1}^{y} e^{i H_{\sigma} t} e^{-i H_{\mathrm{eff}}^{\prime} t} \sigma_{1}^{y},
$$

where $t=\pi /\left(4 \sqrt{\alpha^{2}+\kappa^{2}}\right)$. When $i$ SWAP is available, the CNOT gate can be constructed in the standard way [35]

$$
\begin{aligned}
U_{\mathrm{CNOT}}= & e^{-i(\pi / 4) \sigma_{1}^{z}} e^{i(\pi / 4) \sigma_{2}^{x}} e^{i(\pi / 4) \sigma_{2}^{z}} \\
& \times U_{i \mathrm{SWAP}} e^{-i(\pi / 4) \sigma_{1}^{x}} U_{i \mathrm{SWAP}} e^{i(\pi / 4) \sigma_{2}^{z}} .
\end{aligned}
$$

Since $H_{\text {eff }}^{\prime}$ is an approximation of $H_{\text {eff }}$, the above sequence will yield an approximate CNOT $U_{\mathrm{CNOT}}^{\prime}$ when implemented with the full the Hamiltonian. The success of the sequence therefore depends on the fidelity of the gate $F\left(U_{\mathrm{CNOT}}^{\prime}\right)$. Ideally, the fidelity would be defined using a minimization over all possible states of two qubits. However, to characterize the fidelity of an imperfect CNOT, it is sufficient to consider the following four logical states of two qubits [23]: $|+\uparrow\rangle,|+\downarrow\rangle,|-\uparrow\rangle$, and $|-\downarrow\rangle$. These four states are product states that, when acted upon by a perfect CNOT, become the four maximally entangled Bell states $\left|\Phi^{+}\right\rangle,\left|\Psi^{+}\right\rangle,\left|\Phi^{-}\right\rangle$, and $\left|\Psi^{-}\right\rangle$, respectively. As such, the fidelity of an imperfect CNOT may be defined as

$$
F\left(U_{\mathrm{CNOT}}^{\prime}\right)=\min _{i \in\{+,-\}, j \in\{0,1\}}\left|\left\langle i j\left|U_{\mathrm{CNOT}}^{\dagger} U_{\mathrm{CNOT}}^{\prime}\right| i j\right\rangle\right|^{2} .
$$

The choice of basis used here ensures that $F\left(U_{\mathrm{CNOT}}^{\prime}\right)$ gives a good characterization of the properties of $U_{\mathrm{CNOT}}^{\prime}$ in comparison to a perfect CNOT, especially for the required task of generating entanglement. For realistic parameters, with the Zeeman terms 2 orders of magnitude stronger than the qubit-qubit coupling, the above sequence yields a fidelity for the CNOT gate of $99.976 \%$.

To compare these values to the thresholds found in schemes for quantum computation, we must first note that imperfect CNOTs in these cases are usually modeled by the perfect implementation of the gate followed by depolarizing noise at a certain probability. It is known that such noisy CNOTs can be used for quantum computation in the surface code if the depolarizing probability is less than
$1.1 \%$ [36]. Such probability corresponds to a fidelity, according to the definition above, of $99.17 \%$. The fidelities that may be achieved in the schemes proposed here are well above this value, and hence, although they do not correspond to the same noise model, we can expect these gates to be equally suitable for fault-tolerant quantum computation.

\section{DECOHERENCE}

In this section, we study the dynamics of a single qubit coupled to the ferromagnet. In particular, we want to answer the question of whether the two-qubit dynamics studied in the previous section is coherent, i.e., whether the decoherence time solely due to the dipolar coupling to the ferromagnet is larger than the qubit operation time.

A ferromagnet has two types of fluctuations-longitudinal and transverse. The longitudinal noise stems from fluctuations of the longitudinal $S^{\tilde{z}}$ component (we recall that the ferromagnet is polarized along $\tilde{z}$ ), while the transverse one is related to fluctuations of $S^{\tilde{x}}$. In what follows, we study these two noise sources separately. The general noise model that describes both types of noise is then given by

$$
H=H_{F}+\frac{\Delta}{2} \sigma^{z}+\sigma^{z} \otimes X+\sigma^{+} \otimes Y+\text { H.c., }
$$

where the ferromagnet operators $X(Y)$ with zero expectation value couple longitudinally (transversally) to the qubit. The noise model given in Eq. (15) leads to the following relaxation and decoherence times within the Born-Markov approximation [37]:

$$
\begin{gathered}
T_{1}^{-1}=S_{Y}(\omega=\Delta), \\
T_{2}^{-1}=\frac{1}{2} T_{1}^{-1}+S_{X}(\omega=0),
\end{gathered}
$$

where we define the fluctuation power spectrum of an operator $A$ in the following way: $S_{A}(\omega)=$ $\int d t e^{-i \omega t}\left\{A^{\dagger}(t), A(0)\right\}$. In order to obtain an expression for the decoherence times, we need a specific model for the ferromagnet Hamiltonian, herein taken to be a gapped Heisenberg model $H_{F}=-J \sum_{\left\langle\mathbf{r}, \mathbf{r}^{\prime}\right\rangle} S_{\mathbf{r}} \cdot S_{\mathbf{r}^{\prime}}+\Delta_{F} \sum_{\mathbf{r}} S_{\mathbf{r}}^{z}, J$ being the exchange coupling and $\Delta_{F}$ the excitation gap.

\section{A. Longitudinal noise}

The power spectrum of longitudinal fluctuations is given by the following expression for $\omega, \Delta_{F} \gg T$ [see Appendix $\mathrm{C}$ and, in particular, Eq. (C8)]:

$$
S_{\|}^{3 \mathrm{D}}(\omega)=\frac{\zeta \sqrt{\beta \omega}}{2 \beta^{2} D^{3}} e^{-\beta \Delta_{F}},
$$

where $\zeta=\sqrt{\pi}-e \pi \operatorname{Erfc}(1)$. For $\omega \ll T$ and $T \ll \Delta_{F}$ [see Appendix C, Eq. (C10)],

$$
S_{\|}^{3 \mathrm{D}}(\omega)=\frac{\exp \left(-\beta \Delta_{F}\right)}{8 \pi \beta^{2} D^{3}},
$$

where $D=2 J S$. We study transverse $(Y)$ and longitudinal $(X)$ couplings separately. In the case of longitudinal 
coupling, we solve the problem exactly, while we treat the transverse coupling within the framework of perturbation theory.

\section{Transverse coupling to longitudinal noise}

The part of the Hamiltonian that describes transverse coupling to the longitudinal noise reads

$$
H=H_{F}+\frac{M_{s}}{S} \sigma_{1}^{+} \otimes \int d \mathbf{r} b_{1, \mathbf{r}} S_{\mathbf{r}}^{\tilde{z}}+\text { H.c. }
$$

Using Eq. (17) and the inequality

$$
S_{\|}^{3 \mathrm{D}}(\omega, \mathbf{r}) \leq S_{\|}^{3 \mathrm{D}}(\omega, \mathbf{r}=0),
$$

we obtain the relaxation time

$$
\begin{aligned}
T_{1}^{-1} & =\frac{M_{s}^{2}}{S^{2}} \int d \mathbf{r} d \mathbf{r}^{\prime} b_{1, \mathbf{r}} b_{1, \mathbf{r}^{\prime}} S_{\|}^{3 \mathrm{D}}\left(\Delta, \mathbf{r}-\mathbf{r}^{\prime}\right) \\
& \leq \frac{M_{s}^{2}}{S^{2}} \int d \mathbf{r} d \mathbf{r}^{\prime} b_{1, \mathbf{r}} b_{1, \mathbf{r}^{\prime}} S_{\|}^{3 \mathrm{D}}(\Delta, \mathbf{r}=0) \\
& =\frac{M_{s}^{2}}{S^{2}} b_{1}^{2} S_{\|}^{3 \mathrm{D}}(\Delta),
\end{aligned}
$$

where the expression for $S_{\|}^{3 \mathrm{D}}(\Delta)$ is given in Eq. (18) since $\Delta \gg T$. The above expression readily shows that relaxation time can be tailored to be arbitrarily small by choosing the ratio $T / \Delta_{F}$ to be sufficiently small.

\section{Longitudinal coupling to longitudinal noise}

Here, we consider only longitudinal coupling to longitudinal noise; thus, the Hamiltonian reads

$$
H=H_{F}+\sigma^{z} \otimes V+\frac{\Delta}{2} \sigma_{z},
$$

with $\Delta$ the qubit splitting and $V=\frac{M_{s}}{S} \int d \mathbf{r} a_{1, \mathbf{r}} S_{\mathbf{r}}^{\tilde{z}}$. To simplify the problem further [38], we substitute $S_{\mathbf{r}}^{\tilde{z}} \rightarrow S_{\mathbf{r}}^{\tilde{x}}$, since $S_{\mathbf{r}}^{\tilde{x}}$ is linear in magnon operators while $S_{\mathbf{r}}^{\tilde{z}}$ is quadratic. When the final formula for the decoherence time is obtained, we substitute back the power spectrum of $S_{\mathbf{r}}^{\tilde{z}}$ instead of $S_{\mathbf{r}}^{\tilde{x}}$.

In order to study decoherence, we have to calculate the following quantity [38]:

$$
\begin{aligned}
\left\langle\sigma^{-}(t)\right\rangle= & e^{i \Delta t}\left\langle\sigma^{-}(0)\right\rangle \\
& \times\left\langle\tilde{T} \exp \left(i \int_{0}^{t} V d t^{\prime}\right) T \exp \left(i \int_{0}^{t} V d t^{\prime}\right)\right\rangle,
\end{aligned}
$$

with $(\tilde{T}) T$ the (anti-) time-ordering operator. The average in the above expression can be evaluated using a cluster expansion [39], and since the perturbation $V$ is linear in the bosonic operators, only the second-order cluster contributes. Therefore, the final exact result for the time evolution of $\sigma^{-}(t)$ reads

$$
\left\langle\sigma^{-}(t)\right\rangle=e^{i \Delta t}\left\langle\sigma^{-}(0)\right\rangle e^{-1 / 2 \int_{0}^{t} \int_{0}^{t} S\left(t_{2}-t_{1}\right) d t_{1} d t_{2}},
$$

where $S(t)=\left\langle[V(t), V(0)]_{+}\right\rangle$. After performing the Fourier transformation, we obtain

$$
\left\langle\sigma_{-}(t)\right\rangle=e^{i \Delta t}\left\langle\sigma_{-}(0)\right\rangle \times \exp \left(-\frac{1}{2} \int \frac{d \omega}{2 \pi} S(\omega) \frac{\sin ^{2}(\omega t / 2)}{(\omega / 2)^{2}}\right) .
$$

Note that this expression is of exactly the same form as the one for a classical Gaussian noise [40]. Now, we substitute back $S_{\mathbf{r}}^{\tilde{x}} \rightarrow S_{\mathbf{r}}^{\tilde{z}}$ :

$$
\begin{aligned}
\left\langle\sigma^{-}(t)\right\rangle= & e^{i \Delta t}\left\langle\sigma^{-}(0)\right\rangle \times \exp \left(-\frac{M_{s}^{2}}{2 S^{2}} \int \frac{d \omega}{2 \pi}\right. \\
& \left.\times \int d \mathbf{r} d \mathbf{r}^{\prime} a_{1, \mathbf{r}} a_{1, \mathbf{r}^{\prime}} S_{\|}^{3 \mathrm{D}}\left(\omega, \mathbf{r}-\mathbf{r}^{\prime}\right) \frac{\sin ^{2}(\omega t / 2)}{(\omega / 2)^{2}}\right) .
\end{aligned}
$$

For long times $t \gg \hbar / T$, the dynamics is of the form

$$
\left\langle\sigma^{-}(t)\right\rangle \sim e^{-a_{1} M_{s}^{2} T^{2} e^{-\beta \Delta_{F} t /\left(8 \pi D^{3} S^{2}\right)+i \Delta t},}
$$

where we have used the inequality $S_{\|}^{3 \mathrm{D}}(\omega, \mathbf{r}) \leq S_{\|}^{3 \mathrm{D}}(\omega, \mathbf{r}=0)$. Thus, this type of decoherence can be suppressed by choosing the ratio $T / \Delta_{F}$ to be sufficiently small.

\section{B. Transverse noise}

The power spectrum of transverse fluctuations of the ferromagnet is gapped and thus vanishes for $\omega<\Delta_{F}$ [see Eqs. (B19) and (B23) in Appendix B]:

$$
S_{\perp}^{3 \mathrm{D}}(\omega)=0, \quad \omega<\Delta_{F},
$$

$S_{\perp}^{3 \mathrm{D}}(\omega)=\frac{S \sqrt{\omega-\Delta_{F}}}{D^{3 / 2}} \operatorname{coth}(\beta \omega / 2), \quad \omega>\Delta_{F}$.

Since the transverse fluctuations are gapped and the precession frequency of the qubits is below the gap, this noise source does not contribute in the second order (Born approximation) because only virtual magnons can be excited. In this section, we choose the quantization axes such that qubit splitting is along the $z$ axis, while the ferromagnet is polarized along the $x$ axis (see Fig. 1); such a choice of the axis is assumed solely for simplicity, and all the conclusions are also valid for the most general case. The Hamiltonian of the coupled system is of the form of Eq. (15) with operators $X(Y)$ :

$$
\begin{gathered}
X=\frac{i M_{s}}{2 S} \int d \mathbf{r} c_{\mathbf{r}}\left(S_{\mathbf{r}}^{+}-S_{\mathbf{r}}^{-}\right), \\
Y^{+}=-\frac{i M_{s}}{8 S} \int d \mathbf{r}\left(a_{\mathbf{r}} S_{\mathbf{r}}^{+}+b_{\mathbf{r}} S_{\mathbf{r}}^{-}\right),
\end{gathered}
$$

with $S_{\mathbf{r}}^{ \pm}=S_{\mathbf{r}}^{y} \pm i S_{\mathbf{r}}^{z}$ and the definitions

$$
a_{\mathbf{r}}=B_{\mathbf{r}}+3 C_{\mathbf{r}}-6 A_{\mathbf{r}} \text {, }
$$




$$
\begin{gathered}
b_{\mathbf{r}}=B_{\mathbf{r}}+3 C_{\mathbf{r}}+6 A_{\mathbf{r}}, \\
c_{\mathbf{r}}=B_{\mathbf{r}}-3 A_{\mathbf{r}}^{\prime \prime},
\end{gathered}
$$

where $A_{\mathbf{r}}, B_{\mathbf{r}}$, and $C_{\mathbf{r}}$ are given by Eqs. (3)-(5). To proceed further, we perform the SW transformation on the Hamiltonian given by Eq. (15). We ignore the Lamb and Stark shifts and obtain the effective Hamiltonian

$$
H=H_{F}+\frac{\Delta}{2} \sigma^{z}+\sigma^{z} \otimes \tilde{X}_{2}+\sigma^{+} \otimes \tilde{Y}_{2}^{-}+\sigma^{-} \otimes \tilde{Y}_{2}^{+},
$$

where

$$
\begin{gathered}
\tilde{X}_{2}=X_{2}-\left\langle X_{2}\right\rangle, \\
\tilde{Y}_{2}^{ \pm}=Y_{2}^{ \pm}-\left\langle Y_{2}^{ \pm}\right\rangle,
\end{gathered}
$$

with the following notation:

$$
\begin{gathered}
X_{2}=4\left(Y_{\Delta}^{+} Y^{-}+Y^{+} Y_{\Delta}^{-}\right), \\
Y_{2}^{+}=2\left(Y_{\Delta}^{+} X-X_{0} Y^{+}\right), \\
X_{\omega}=\frac{i M_{s}}{2 S} \int d \mathbf{r} d \mathbf{r}^{\prime} \chi_{\perp}\left(\omega, \mathbf{r}-\mathbf{r}^{\prime}\right) c_{\mathbf{r}}\left(S_{\mathbf{r}^{\prime}}^{+}-S_{\mathbf{r}^{\prime}}^{-}\right), \\
Y_{\omega}^{+}=-\frac{i M_{s}}{8 S} \int d \mathbf{r} d \mathbf{r}^{\prime} \chi_{\perp}\left(\omega, \mathbf{r}-\mathbf{r}^{\prime}\right)\left(a_{\mathbf{r}^{\prime}} S_{\mathbf{r}^{\prime}}^{+}+b_{\mathbf{r}} S_{\mathbf{r}^{\prime}}^{-}\right),
\end{gathered}
$$

The model given by Eq. (15) yields the following expressions for the relaxation and decoherence times:

$$
\begin{gathered}
T_{1}^{-1}=S_{\tilde{Y}_{2}^{-}}(\omega=\Delta), \\
T_{2}^{-1}=\frac{1}{2} T_{1}^{-1}+S_{\tilde{X}_{2}}(\omega=0) .
\end{gathered}
$$

After a lengthy calculation, we obtain the following expressions for $T_{1}$ and $T_{2}$ (see Appendix $\mathrm{E}$ for a detailed derivation):

$$
\begin{aligned}
T_{1}^{-1} & \leq \frac{B^{4} M_{s}^{4} \Delta_{F}^{2}}{2 D^{3} S^{2}}\left(\frac{1}{\Delta_{F}}+\frac{1}{\Delta_{F}-\Delta}\right)^{2} f\left(\frac{\Delta}{\Delta_{F}}, \beta \Delta_{F}\right), \\
T_{2}^{-1} \leq & \frac{B^{4} M_{s}^{4} \Delta_{F}^{2}}{4 D^{3} S^{2}}\left(\frac{1}{\Delta_{F}}+\frac{1}{\Delta_{F}-\Delta}\right)^{2} f\left(\frac{\Delta}{\Delta_{F}}, \beta \Delta_{F}\right) \\
& +\frac{B^{4} M_{s}^{4} \Delta_{F}^{2}}{2 D^{3} S^{2}\left(\Delta_{F}-\Delta\right)^{2}} f\left(0, \beta \Delta_{F}\right),
\end{aligned}
$$

with the function $f(x, y)$ defined as follows:

$$
f(x, y)=\int_{1+x}^{\infty} d z \frac{\sqrt{z-1}}{e^{y z}-1} \frac{\sqrt{z-x-1}}{e^{y(z-x)}-1} .
$$

It is important to note that $f(x, y) \propto e^{-y}$; i.e., we obtain, as for the longitudinal noise, that the effect of transverse fluctuations can be suppressed by choosing the temperature to be much smaller than the excitation gap of the ferromagnet. As anticipated, Eq. (45) shows that the transverse noise becomes more important as the resonance is approached $\left(\Delta \sim \Delta_{F}\right)$.

\section{ESTIMATES}

In this section, we give numerical estimates for the coherent coupling mediated by the ferromagnet and the associated decoherence times. These estimates are valid for both silicon-based and NV-center qubits.

Let us assume that the qubits lie close to the disk axis at a distance $h=25 \mathrm{~nm}$ below the disk and that the ferromagnet has in-plane polarization (along the $x$ axis); for the ferromagnet, we assume yttrium iron garnet (iron), that the thickness of the disk is $20 \mathrm{~nm}$, its radius is $50 \mathrm{~nm}$, its Curie temperature is $550 \mathrm{~K}(1041 \mathrm{~K})$, its saturation magnetization is about $0.2 \mathrm{~T}(2.2 \mathrm{~T})$, and the lattice constant is $12 \AA$ ( $3 \AA$ ). In this case, the stray field reaches values up to $10 \mathrm{mT}$ $(100 \mathrm{mT})$, and such a magnetic field strength is not detrimental since NV centers can tolerate such small fields perpendicular to the polarization axis; stronger magnetic fields would destroy the ability to manipulate NV centers optically [41]. For these cases and when the qubit splitting is brought close to resonance $\Delta_{F}-\Delta \approx 10^{-2} \mu \mathrm{eV}$ $(0.1 \mu \mathrm{eV})$, we obtain operation times on the order of hundreds (tens) of nanoseconds when the qubits are separated by a distance of about $1 \mu \mathrm{m}$. The decoherence times $T_{2}$ depend strongly on the ratio $k_{B} T / \Delta_{F}$, and the additional decoherence source can be made negligible if this ratio is sufficiently small. For a magnon gap $\Delta_{F}=100 \mu \mathrm{eV}$ and a temperature $T=0.1 \mathrm{~K}$, we obtain decoherence times solely due to the coupling to the ferromagnet that are much bigger than the operation times and the typical decoherence times of the qubits.

\section{CONCLUSIONS}

We propose a scheme to coherently couple two atomistic qubits separated over distances on the order of a micron. We present a sequence for the implementation of the entangling CNOT gate and obtain operation times on the order of a few tens of nanoseconds. We show that there is a regime where all fluctuations of the ferromagnet are under control and the induced decoherence is nondetrimental: This regime is reached when the temperature is smaller than the excitation gap of the ferromagnet. The main novel aspect of our proposal is its applicability to the technologically very important silicon qubits and NV centers to which previous coupling methods do not apply.

\section{ACKNOWLEDGMENTS}

We would like to thank A. Yacoby, A. Morello, R. Warburton, and T. van der Sar for useful discussions. This work was supported by SNF, NCCR QSIT, and IARPA.

\section{APPENDIX A: HOLSTEIN-PRIMAKOFF TRANSFORMATION}

For the sake of completeness, we derive in this Appendix explicit expressions for the different spin-spin correlators used in this work: 


$$
C^{\alpha \beta}(\omega, \mathbf{q})=\left\langle S_{\mathbf{q}}^{\alpha}(\omega) S_{-\mathbf{q}}^{\beta}(0)\right\rangle
$$

For this purpose, we make use of a Holstein-Primakoff transformation

$$
\begin{gathered}
S_{i}^{z}=-S+n_{i}, \quad S_{i}^{-}=\sqrt{2 S} \sqrt{1-\frac{n_{i}}{2 S}} a_{i}, \quad \text { and } \\
S_{i}^{+}=\left(S_{i}^{-}\right)^{\dagger},
\end{gathered}
$$

in the limit $n_{i} \ll 2 S$, with $a_{i}$ satisfying bosonic commutation relations and $n_{i}=a_{i}^{\dagger} a_{i}$ [42]. The creation operators $a_{i}^{\dagger}$ and annihilation operators $a_{i}$ satisfy bosonic commutation relations, and the associated particles are called magnons. The corresponding Fourier transforms are straightforwardly defined as $a_{\mathbf{q}}^{\dagger}=\frac{1}{\sqrt{N}} \sum_{i} e^{-i \mathbf{q} \cdot \mathbf{R}_{i}} a_{i}$. In harmonic approximation, the Heisenberg Hamiltonian $H_{F}$ reads

$$
H_{F} \approx \sum_{\mathbf{q}} \epsilon_{\mathbf{q}} a_{\mathbf{q}}^{\dagger} a_{\mathbf{q}}
$$

where $\epsilon_{\mathbf{q}}=\omega_{\mathbf{q}}+\Delta_{F}=4 J S\left\{3-\left[\cos \left(q_{x}\right)+\cos \left(q_{y}\right)+\right.\right.$ $\left.\left.\cos \left(q_{z}\right)\right]\right\}+\Delta_{F}$ is the spectrum for a cubic lattice with lattice constant $a=1$ and the gap $\Delta_{F}$ is induced by the external magnetic field or anisotropy of the ferromagnet.
APPENDIX B: TRANSVERSE CORRELATORS

$$
\left\langle S_{\mathbf{q}}^{+}(\boldsymbol{t}) \boldsymbol{S}_{-\mathbf{q}}^{-}(\mathbf{0})\right\rangle
$$

Let us now define the Fourier transforms in the harmonic approximation

$$
\begin{gathered}
S_{\mathbf{q}}^{+}=\frac{1}{\sqrt{N}} \sum_{i} e^{-i \mathbf{q} \mathbf{r}_{i}} S_{i}^{+}=\frac{\sqrt{2 S}}{\sqrt{N}} \sum_{i} e^{-i \mathbf{q} \mathbf{r}_{i}} a_{i}^{\dagger}=\sqrt{2 S} a_{-\mathbf{q}}^{\dagger}, \\
S_{-\mathbf{q}}^{-}=\frac{1}{\sqrt{N}} \sum_{i} e^{i \mathbf{q} \mathbf{r}_{i}} S_{i}^{-}=\frac{\sqrt{2 S}}{\sqrt{N}} \sum_{i} e^{i \mathbf{q} \mathbf{r}_{i}} a_{i}=\sqrt{2 S} a_{-\mathbf{q}} .
\end{gathered}
$$

From the above equations, it directly follows that

$$
\begin{aligned}
C^{+-}(t, \mathbf{q}) & =\left\langle S_{\mathbf{q}}^{+}(t) S_{-\mathbf{q}}^{-}(0)\right\rangle=2 S\left\langle a_{-\mathbf{q}}^{\dagger}(t) a_{-\mathbf{q}}\right\rangle \\
& =2 S e^{i \epsilon_{\mathbf{q}} t} n_{\mathbf{q}},
\end{aligned}
$$

with $\epsilon_{\mathbf{q}} \approx D q^{2}+\Delta_{F}$ in the harmonic approximation.

The Fourier transform is then simply given by

$$
\begin{aligned}
C^{+-}(\omega, \mathbf{q}) & =\frac{1}{\sqrt{2 \pi}} \int_{-\infty}^{\infty} d t e^{-i \omega t} C^{+-}(t, \mathbf{q}) \\
& =\frac{1}{\sqrt{2 \pi}} \int_{-\infty}^{\infty} d t e^{i\left(\epsilon_{\mathbf{q}}-\omega\right) t} 2 S n_{\mathbf{q}} \\
& =\sqrt{2 \pi} 2 S \delta\left(\epsilon_{\mathbf{q}}-\omega\right) \frac{1}{e^{\beta \omega}-1} .
\end{aligned}
$$

The corresponding correlator in real space is then simply given by $(q:=|\mathbf{q}|)$

$$
\begin{aligned}
C^{+-}(\omega, \mathbf{r}) & =\frac{1}{(2 \pi)^{3 / 2}} \int d \mathbf{q} e^{i \mathbf{q r}} C^{+-}(\omega, \mathbf{q})=\frac{\sqrt{2 \pi}}{(2 \pi)^{3 / 2}} 2 S \frac{1}{e^{\beta \omega}-1} \int d \mathbf{q} \delta\left(D q^{2}+\Delta_{F}-\omega\right) e^{i \mathbf{q r}} \\
& =\frac{2 S}{e^{\beta \omega}-1} \int_{-1}^{1} \int_{0}^{\infty} d q d x q^{2} \delta\left(D q^{2}+\Delta_{F}-\omega\right) e^{i q r x}=\frac{4 S}{r} \frac{1}{e^{\beta \omega}-1} \int_{0}^{\infty} d q q \delta\left(D q^{2}+\Delta_{F}-\omega\right) \sin (q r) .
\end{aligned}
$$

Let us now perform the following substitution:

$$
y=D q^{2}
$$

which gives for $\omega>\Delta_{F}$

$$
C^{+-}(\omega, \mathbf{r})=\frac{4 S / r}{2 D\left(e^{\beta \omega}-1\right)} \int_{0}^{\infty} d y \delta\left(y+\Delta_{F}-\omega\right) \sin \left(\sqrt{\frac{y}{D}} r\right)=\frac{2 S}{D} \frac{1}{e^{\beta \omega}-1} \frac{\sin \left[\sqrt{\left(\omega-\Delta_{F}\right) / D} r\right]}{r} .
$$

We remark that

$$
C^{+-}(\omega, \mathbf{r})=0, \quad \omega<\Delta_{F} .
$$

We note the diverging behavior of the above correlation function for $\Delta_{F}=0$ and $\omega \rightarrow 0$, namely,

$$
\frac{1}{e^{\beta \omega}-1} \frac{\sin \left(\sqrt{\frac{\omega}{D}} r\right)}{r} \rightarrow \frac{1}{\sqrt{D} \beta} \frac{1}{\sqrt{\omega}} .
$$

Similarly, it is now easy to calculate the corresponding commutators and anticommutators. Let us define

$$
S_{\perp}(t, \mathbf{q}):=\frac{1}{2}\left\{S_{\mathbf{q}}^{+}(t), S_{-\mathbf{q}}^{-}(0)\right\} .
$$

It is then straightforward to show that

$$
S_{\perp}(t, \mathbf{q})=S e^{i \epsilon_{\mathbf{q}} t}\left(1+2 n_{\mathbf{q}}\right),
$$

and therefore,

$$
\begin{aligned}
S_{\perp}(\omega, \mathbf{q}) & =\frac{S}{\sqrt{2 \pi}} \int_{-\infty}^{\infty} e^{i\left(\epsilon_{\mathbf{q}}-\omega\right) t}\left(1+2 n_{\mathbf{q}}\right) \\
& =S \sqrt{2 \pi} \delta\left(\epsilon_{\mathbf{q}}-\omega\right)\left(1+2 \frac{1}{e^{\beta \omega}-1}\right) .
\end{aligned}
$$

Following essentially the same steps as the one performed above, we obtain the 3D real-space anticommutator for $\omega>\Delta_{F}$ : 


$$
\begin{aligned}
S_{\perp}^{3 \mathrm{D}}(\omega, \mathbf{q})= & S \operatorname{coth}(\beta \omega / 2) \\
& \times \int_{-1}^{1} \int_{0}^{\infty} d x d q q^{2} e^{i q r x} \delta\left(\epsilon_{\mathbf{q}}-\omega\right) \\
= & \frac{S}{D} \operatorname{coth}(\beta \omega / 2) \frac{\sin \left[\sqrt{\left(\omega-\Delta_{F}\right) / D} r\right]}{r} .
\end{aligned}
$$

Let us now finally calculate the transverse susceptibility defined as

$$
\chi_{\perp}(t, \mathbf{q})=-i \theta(t)\left[S_{\mathbf{q}}^{+}(t), S_{-\mathbf{q}}^{-}(0)\right]
$$

As before, in the harmonic approximation, one finds

$$
\chi_{\perp}(t, \mathbf{q})=i \theta(t) 2 S e^{i \epsilon_{\mathbf{q}} t} .
$$

In the frequency domain, we then have

$$
\begin{aligned}
\chi_{\perp}(\omega, \mathbf{q}) & =\frac{2 i S}{\sqrt{2 \pi}} \int_{0}^{\infty} d t e^{i\left(\epsilon_{\mathbf{q}}-\omega\right) t-\eta t} \\
& =-\frac{2 S}{\sqrt{2 \pi}} \frac{1}{\epsilon_{\mathbf{q}}-\omega+i \eta}
\end{aligned}
$$

and thus, in the small $\mathbf{q}$ expansion,

$$
\chi_{\perp}(\omega, \mathbf{q})=-\frac{2 S}{\sqrt{2 \pi}} \frac{1}{D q^{2}+\Delta_{F}-\omega+i \eta} .
$$

$$
\begin{aligned}
\chi_{\perp}^{3 \mathrm{D}}(\omega, \mathbf{r}) & =-\frac{2 S}{\sqrt{2 \pi}} \frac{2 \pi}{(2 \pi)^{3 / 2}} \int_{0}^{\infty} \int_{-1}^{1} d x d q q^{2} \frac{1}{D q^{2}+\Delta_{F}-\omega+i \eta} e^{i q r x} \\
& =-\frac{4 S}{\sqrt{2 \pi}} \frac{2 \pi}{(2 \pi)^{3 / 2}} \frac{1}{r} \int_{0}^{\infty} d q q \frac{1}{D q^{2}+\Delta_{F}-\omega+i \eta} \sin (q r) .
\end{aligned}
$$

Making use of the Plemelj formula, we obtain for $\omega>\Delta_{F}$

$$
\begin{aligned}
\chi_{\perp}^{3 \mathrm{D}}(\omega, \mathbf{r}) & =-\frac{2 S}{\sqrt{2 \pi}} \frac{2 \pi}{(2 \pi)^{3 / 2}} \frac{1}{r} \int_{-\infty}^{\infty} d q q \frac{1}{D q^{2}+\Delta_{F}-\omega+i \eta} \sin (q r) \\
& =-\frac{2 S}{\sqrt{2 \pi}} \frac{2 \pi}{(2 \pi)^{3 / 2}} \frac{1}{r} P \int_{-\infty}^{\infty} d q \frac{q}{D q^{2}+\Delta_{F}-\omega} \sin (q r)+i \frac{2 S}{\sqrt{2 \pi}} \frac{2 \pi^{2}}{(2 \pi)^{3 / 2}} \frac{1}{r} \int_{-\infty}^{\infty} d q q \delta\left(D q^{2}+\Delta_{F}-\omega\right) \sin (q r) \\
& =-\frac{S}{D} \frac{\cos \left[r \sqrt{\left(\omega-\Delta_{F}\right) / D}\right]}{r}+i \frac{S}{2 D} \frac{\sin \left[\sqrt{\left(\omega-\Delta_{F}\right) / D} r\right]}{r}
\end{aligned}
$$

It is worth pointing out that the imaginary part of the susceptibility vanishes

$$
\chi_{\perp}^{3 \mathrm{D}}(\omega, \mathbf{r})^{\prime \prime}=0, \quad \omega<\Delta_{F},
$$

and therefore, the susceptibility is purely real and takes the form of a Yukawa potential:

$$
\chi_{\perp}^{3 \mathrm{D}}(\omega, \mathbf{r})=-\frac{S}{D} \frac{e^{-r / l_{F}}}{r}, \quad \omega<\Delta_{F},
$$

where

$$
l_{F}=\sqrt{\frac{D}{\Delta_{F}-\omega}} .
$$

Note also that the imaginary part of the transverse susceptibility satisfies the well-known fluctuation-dissipation theorem

$$
S_{\perp}^{3 \mathrm{D}}(\omega, \mathbf{r})=\operatorname{coth}(\beta \omega / 2) \chi_{\perp}^{3 \mathrm{D}}(\omega, \mathbf{r})^{\prime \prime} .
$$

In three dimensions, the susceptibility decays as $1 / r$, where $r$ is measured in lattice constants. For distances of the order of $1 \mu \mathrm{m}$, this decay leads to a reduction of 4 orders of magnitude.
For quasi-one-dimensional ferromagnets, such a reduction is absent and the transverse susceptibility reads

$$
\chi_{\perp}^{1 \mathrm{D}}(\omega, r)=-\frac{S}{D} l_{F} e^{-r / l_{F}}, \quad \omega<\Delta_{F},
$$

where $l_{F}$ is defined as above and the imaginary part vanishes as above, i.e.,

$$
\chi_{\perp}^{1 \mathrm{D}}(\omega, r)^{\prime \prime}=0, \quad \omega<\Delta_{F} .
$$

Similarly, for $\omega>\Delta_{F}$, we have

$$
\chi_{\perp}^{1 \mathrm{D}}(\omega, r)=S \frac{\sin \left[\sqrt{\left(\omega-\Delta_{F}\right) / D} r\right]}{\sqrt{D\left(\omega-\Delta_{F}\right)}}
$$

and

$\chi_{\perp}^{1 \mathrm{D}}(\omega, r)^{\prime \prime}=\frac{S}{2 D} \sqrt{\frac{D}{\omega-\Delta_{F}}} \cos \left[\sqrt{\left(\omega-\Delta_{F}\right) / D} r\right]$. 
APPENDIX C: LONGITUDINAL CORRELATORS

$$
\left\langle\boldsymbol{S}_{\mathbf{q}}^{z}(t) S_{-\mathbf{q}}^{z}(\mathbf{0})\right\rangle
$$

The longitudinal susceptibility reads

$$
\begin{aligned}
\chi_{\|}(t, \mathbf{q}) & =-i \theta(t)\left[S_{\mathbf{q}}^{z}(t), S_{-\mathbf{q}}^{z}(0)\right] \\
& =-\theta(t) \frac{1}{N} \sum_{\mathbf{q}^{\prime}, \mathbf{q}^{\prime \prime}} e^{i t\left(\epsilon_{\mathbf{q}^{\prime}}-\epsilon_{\mathbf{q}^{\prime}+\mathbf{q}}\right)}\left\langle\left[a_{\mathbf{q}^{\prime}}^{\dagger} a_{\mathbf{q}^{\prime}+\mathbf{q}}, a_{\mathbf{q}^{\prime \prime}}^{\dagger} a_{\mathbf{q}^{\prime \prime}-\mathbf{q}}\right]\right\rangle .
\end{aligned}
$$

Applying Wick's theorem and performing a Fourier transform, we obtain the susceptibility in the frequency domain

$$
\chi_{\|}(\omega, \mathbf{q})=-\frac{1}{N} \sum_{\mathbf{k}} \frac{n_{\mathbf{k}}-n_{\mathbf{k}+\mathbf{q}}}{\omega-\epsilon_{\mathbf{k}+\mathbf{q}}+\epsilon_{\mathbf{k}}+i \eta},
$$

where $n_{\mathbf{k}}$ is the magnon occupation number given by the Bose-Einstein distribution

$$
n_{k}=\frac{1}{e^{\beta \epsilon_{\mathrm{k}}}-1},
$$

where $\epsilon_{\mathbf{k}}$ is again the magnon spectrum $\left(\epsilon_{\mathbf{k}}=\right.$ $\omega_{\mathbf{k}}+\Delta_{F} \approx D k^{2}+\Delta_{F}$ for small $k$ ). Note that the longitudinal susceptibility is proportional to $1 / S$, due to the fact that $\epsilon_{\mathbf{k}}-\epsilon_{\mathbf{k}+\mathbf{q}}=\omega_{\mathbf{k}}-\omega_{\mathbf{k}+\mathbf{q}} \propto S$.

Since we are interested in the decoherence processes caused by the longitudinal fluctuations, we calculate the imaginary part of $\chi_{\|}(\omega, \mathbf{q})$ that is related to the fluctuations via the fluctuation-dissipation theorem. Performing a small q expansion and assuming without loss of generality $\omega>0$, we obtain for the imaginary part

$$
\begin{aligned}
\chi_{\|}^{3 \mathrm{D}}(\omega, \mathbf{q})^{\prime \prime} & =\frac{\pi}{(2 \pi)^{3}} \int d \mathbf{k}\left(n_{\mathbf{k}}-n_{\mathbf{k}+\mathbf{q}}\right) \delta\left(\omega_{\mathbf{k}}-\omega_{\mathbf{k}+\mathbf{q}}+\omega\right) \\
& =\frac{1}{4 \pi} \int_{0}^{\infty} d k k^{2} \int_{-1}^{1} d x\left(\frac{1}{e^{\beta\left(\Delta_{F}+D k^{2}\right)}-1}-\frac{1}{e^{\beta\left(\omega+\Delta_{F}+D k^{2}\right)}-1}\right) \delta\left(\omega-D q^{2}-2 D k q x\right) \\
& =\frac{1}{4 \pi} \int_{0}^{\infty} d k k^{2} \int_{-1}^{1} d x\left(\frac{1}{e^{\beta\left(\Delta_{F}+D k^{2}\right)}-1}-\frac{1}{e^{\beta\left(\omega+\Delta_{F}+D k^{2}\right)}-1}\right) \delta\left(k-\frac{\omega-D q^{2}}{2 D q x}\right)\left|\frac{1}{2 D q x}\right| \\
& =\frac{1}{4 \pi} \int_{-1}^{1} d x\left|\frac{1}{2 D q x}\right|\left(\frac{\omega-D q^{2}}{2 D q x}\right)^{2}\left(\frac{1}{e^{\beta\left[\Delta_{F}+D\left(\frac{\omega-D q^{2}}{2 D q x}\right)^{2}\right]}-1}-\frac{1}{e^{\beta\left[\omega+\Delta_{F}+D\left(\frac{\omega-D q^{2}}{2 D q x}\right)^{2}\right]}-1}\right) \theta\left(\frac{\omega-D q^{2}}{2 D q x}\right) \\
& =\frac{1}{4 \pi} \int_{0}^{1} d x \frac{1}{2 D q x}\left(\frac{\omega-D q^{2}}{2 D q x}\right)^{2}\left(\frac{1}{e^{\beta\left[\Delta_{F}+D\left(\frac{\omega-D q^{2}}{2 D q x}\right)^{2}\right]}-1}-\frac{1}{e^{\beta\left[\omega+\Delta_{F}+D\left(\frac{\omega-D q^{2}}{2 D q x}\right)^{2}\right]}-1}\right) .
\end{aligned}
$$

Let us first consider the regime where $\omega \gg T$ (and thus $\beta \omega \gg 1$ ); thus, we have $n_{\mathbf{k}} \gg n_{\mathbf{k}+\mathbf{q}}$. Furthermore, we approximate the distribution function $n_{\mathbf{k}}=\frac{e^{-\beta\left(\Delta_{F}+\omega_{\mathbf{k}}\right)}}{1-e^{-\beta \Delta_{F}}+\beta \omega_{\mathbf{k}}}$ (this approximation is valid when $\left.\beta \omega_{\mathbf{k}} \ll 1\right)$ and arrive at the following expression:

$$
\begin{aligned}
\chi_{\|}^{3 \mathrm{D}}(\omega, \mathbf{q})^{\prime \prime} & =\frac{1}{4 \pi} \int_{0}^{1} d x \frac{1}{2 D q x}\left(\frac{\omega-D q^{2}}{2 D q x}\right)^{2} \frac{e^{-\beta\left[\Delta_{F}+D\left(\frac{\omega-D q^{2}}{2 D q x}\right)^{2}\right]}}{1-e^{-\beta \Delta_{F}}+\beta D\left(\frac{\omega-D q^{2}}{2 D q x}\right)^{2}} \\
& =-\frac{e^{1-e^{-\beta \Delta_{F}-\beta \Delta_{F}}}}{4 \beta D^{2} q} \operatorname{Ei}\left[e^{-\beta \Delta_{F}}+\frac{1}{4}\left(-4-\beta D q^{2}+2 \beta \omega-\frac{\beta \omega^{2}}{D q^{2}}\right)\right],
\end{aligned}
$$

where $\operatorname{Ei}(z)$ is the exponential integral function. We also need the real-space representation obtained after inverse Fourier transformation

$$
\chi_{\|}^{3 \mathrm{D}}(\omega, \mathbf{r})^{\prime \prime}=\sqrt{\frac{2}{\pi}} \frac{1}{r} \int_{0}^{\infty} d q q \chi_{\|}^{3 \mathrm{D}}(\omega, q)^{\prime \prime} \sin (q r)
$$

In order to perform the above integral, we note that the imaginary part of the longitudinal susceptibility, given by Eq. (C5), is peaked around $q=\sqrt{\omega / D}$, with the width of the peak $(1 / \sqrt{\beta D})$ much smaller than its position in the regime in which we are working $(\omega \gg T)$. For $\mathbf{r}=\mathbf{0}$, the integration over $q$ can then be performed approximately and yields the following expression:

$$
\chi_{\|}^{3 \mathrm{D}}(\omega, \mathbf{r}=\mathbf{0})^{\prime \prime}=\frac{\sqrt{\pi} e^{-e^{-\beta \Delta_{F}-3 \beta \Delta_{F} / 2}}}{2 \beta^{2} D^{3}}\left[e^{e^{-\beta \Delta_{F}+\beta \Delta_{F} / 2}}-e \sqrt{\pi} \sqrt{e^{\beta \Delta_{F}}-1} \operatorname{Erfc}\left(e^{-\beta \Delta_{F} / 2} \sqrt{e^{\beta \Delta_{F}}-1}\right)\right] \sqrt{\beta \omega},
$$

where $\operatorname{Erfc}(z)$ denotes the complementary error function. It is readily observed from the above expression that the longitudinal fluctuations are exponentially suppressed by the gap. Assuming that $\Delta_{F} \gg T$, we obtain the following simplified expression: 


$$
\chi_{\|}^{3 \mathrm{D}}(\omega, \mathbf{r}=\mathbf{0})^{\prime \prime}=\frac{\sqrt{\pi}-e \pi \operatorname{Erfc}(1)}{2 \beta^{2} D^{3}} e^{-\beta \Delta_{F}} \sqrt{\beta \omega} .
$$

Next, consider the behavior of the longitudinal susceptibility in the opposite limit, when $\beta \omega \ll 1$. In this limit, the difference of the two Boltzmann factors in Eq. (C4) can be expanded to the lowest order in the small quantity $\beta \omega$ :

$$
\chi_{\|}^{3 \mathrm{D}}(\omega, \mathbf{q})^{\prime \prime}=\int_{0}^{1} d x \frac{1}{8 \pi D q x}\left(\frac{\omega-D q^{2}}{2 D q x}\right)^{2} \frac{\beta \omega}{\operatorname{ch}\left[\beta \Delta_{F}+\beta D\left(\frac{\omega-D q^{2}}{2 D q x}\right)^{2}\right]-1}=\frac{\omega}{16 \pi D^{2} q\left(e^{\beta \Delta_{F}+\frac{\beta\left(\omega-D q^{2}\right)^{2}}{4 D q^{2}}}-1\right)} .
$$

In order to calculate the Fourier transform to real space, we note that for $\omega \ll \Delta_{F}$, the denominator of the above expression depends only weakly on $\omega$; thus, we ignore this dependence and obtain the Fourier transform for $\mathbf{r}=0$ :

$$
\chi_{\|}^{3 \mathrm{D}}(\omega)^{\prime \prime}=\frac{\ln \left(1+n_{\mathbf{k}=0}\right)}{16 \pi \beta D^{3}} \omega
$$

From the above equation, we infer that the longitudinal noise of the ferromagnet behaves as an Ohmic bath.

Next, we calculate the longitudinal fluctuations for the case of a quasi-one-dimensional ferromagnet $\left(\Delta_{F} \gg T\right)$ and obtain

$$
\begin{aligned}
\chi_{\|}^{1 \mathrm{D}(\omega, r=0)^{\prime \prime}} & =\frac{1}{4 \pi} \int_{-\infty}^{\infty} d k \int_{-\infty}^{\infty} d q\left(\frac{1}{e^{\beta\left(\Delta_{F}+D k^{2}\right)}-1}-\frac{1}{e^{\beta\left(\omega+\Delta_{F}+D k^{2}\right)}-1}\right) \delta\left(\omega-D q^{2}-2 D k q\right) \\
& =\int_{-\infty}^{\infty} d k \frac{e^{-\beta D k^{2}}}{1-e^{-\beta \Delta_{F}}+\beta D k^{2}} \frac{1}{D \sqrt{k^{2}+\omega / D}}=\frac{\gamma}{D \sqrt{\beta \omega}} e^{-\beta \Delta_{F}}
\end{aligned}
$$

where $\gamma$ is a numerical factor of order unity.

Note that $S_{\|}(\omega, \mathbf{r})$ is defined through the fluctuationdissipation theorem as

$$
S_{\|}(\omega, \mathbf{r})=\operatorname{coth}(\beta \omega / 2) \chi_{\|}(\omega, \mathbf{r})^{\prime \prime} .
$$

\section{APPENDIX D: EXCHANGE COUPLING TO THE FERROMAGNET}

\section{Exchange coupling}

The Hamiltonian we consider is of the following form:

$$
H=H_{F}+H_{\sigma}+A \sum_{i} \boldsymbol{\sigma}_{i} \cdot S_{\mathbf{r}_{i}},
$$

where $A$ is the exchange-coupling constant between the qubit spins and the ferromagnet. The ferromagnet is assumed to be below the Curie temperature, with the magnetization pointing along the out-of-plane $z$ direction. The qubit Hamiltonian reads

$$
H_{\sigma}=\frac{1}{2} \Delta \sum_{i} \sigma_{i}^{x}
$$

\section{Coherent coupling}

We proceed with the derivation of an effective twospin interaction Hamiltonian for $A \ll J$ by employing a perturbative Schrieffer-Wolff transformation [28] up to the second order
$H_{\text {eff }}=H_{\sigma}+\frac{A^{2}}{8} \chi_{\perp}(\Delta)\left(2 \sigma_{1}^{y} \sigma_{2}^{y}+\sigma_{1}^{z} \sigma_{2}^{x}+\sigma_{1}^{x} \sigma_{2}^{z}\right)$,

where we introduce the notation $\chi_{\perp}(\omega)=\chi_{\perp}(\omega, L)$ $\left(L=\left|\mathbf{r}_{2}-\mathbf{r}_{1}\right|\right)$ and $\chi_{\perp}(\omega, \mathbf{r})$ is the transverse real-space spin susceptibility of the ferromagnet. Note that we have neglected $\chi_{\perp}^{3 \mathrm{D}}(-\Delta)$ and $\chi_{\perp}^{3 \mathrm{D}}(0)$ in comparison to $\chi_{\perp}^{1 \mathrm{D}}(\Delta)$, as well as the longitudinal susceptibility $\chi_{\|}$, since it is suppressed by temperature. The real-space transverse susceptibility of the $3 \mathrm{D}$ ferromagnet is given by

$$
\chi_{\perp}^{3 \mathrm{D}}(\omega, \mathbf{r})=-\frac{S}{D} \frac{e^{-r / l_{F}}}{r}, \quad \omega<\Delta_{F},
$$

where $\Delta_{F}$ is the gap induced via an applied external magnetic field or due to internal anisotropy of the ferromagnet $l_{F}=\sqrt{\frac{D}{\Delta_{F}-\omega}}$ and $D=2 J S$. In what follows, we assume that the external gap is always larger than the qubit splitting $\Delta<\Delta_{F}$, as this condition ensures that the transverse noise is not contributing to decoherence in second order since transverse noise is related to the vanishing imaginary part of the transverse susceptibility $\chi_{\perp}(\omega)^{\prime \prime}=0\left(\omega<\Delta_{F}\right)$. The spatial dependence of the effective two-spin coupling given by Eq. (D4) is of Yukawa type because of the presence of the external gap. If we assume a realistic tunnel coupling to the ferromagnet of $100 \mu \mathrm{eV}[43,44]$, the Curie temperature of $550 \mathrm{~K}$ (as, for example, for yttrium iron garnet) and a gap of $\Delta_{F}=100 \mu \mathrm{eV}$, and the qubit splitting close to the resonance $\Delta_{F}-\Delta=3 \times 10^{-3} \mu \mathrm{eV}$ (corresponding to a magnetic field of about $B=60 \mu \mathrm{T}$ ), we obtain for the qubit-qubit-coupling strength a value on the order of 
$4 \times 10^{-11} \mathrm{eV}$ for a lattice constant of about $4 \AA$. This coupling strength gives rise to the operation times of $50 \mu \mathrm{s}$ - significantly below the relaxation and decoherence times of the spin qubit $T_{1}=1 \mathrm{~s}$ [45] and $T_{2}>200 \mu \mathrm{s}$ [46], respectively. Furthermore, the error threshold-defined as the ratio between the two-qubit gate-operation time to the decoherence time-we obtain with such an operation time is about $10^{-2}$, which is good enough for implementing the surface-code error correction [47]. Here, we use $T_{2}$ instead of $T_{2}^{*}$ since spin echo can be performed together with two-qubit gates [48]. Alternatively, the decoherence time of GaAs qubits can be increased without spin echo by narrowing the state of the nuclear spins $[49,50]$.

The dimensionality of the ferromagnet plays an important role-if we assume a 10-nm width of the trench where the ferromagnet is placed, then, for energies below $0.1 \mathrm{meV}$, the ferromagnet behaves quasi-one-dimensionally (1D). In this case, we obtain

$$
\chi_{\perp}^{1 \mathrm{D}}(\omega, r)=-\frac{S}{D} l_{F} e^{-r / l_{F}}, \quad \omega<\Delta_{F},
$$

from where it is evident that at distances $r \lesssim l_{F}$, the susceptibility of a quasi-1D ferromagnet is practically constant, in contrast to the $3 \mathrm{D}$ case, where a $1 / r$ decay is obtained; see Eq. (D4). Additionally, we require $l_{F} \lesssim$ $D /(A S)=2 J / A$ for the perturbation theory to be valid. Thus, for the same parameters as above, but without the need to tune very close to the resonance (we set herein $\Delta_{F}-\Delta=0.5 \mu \mathrm{eV}$, corresponding to about $B=10 \mathrm{mT}$ ), a coupling strength of $10^{-8} \mathrm{eV}$ is obtained.

For the $1 \mathrm{D}$ case, there is yet another rather promising possibility-to use magnetic semiconductors [51]. These materials are characterized by a particularly low Curie temperature of $30 \mathrm{~K}$ or below [51], and the distance between the ions that are magnetically ordered via Ruderman-Kittel-Kasuya-Yosida interaction is about $10-100 \mathrm{~nm}$. Such a large lattice constant is very beneficial for long-range coupling - if we take the lattice constant to be $10 \mathrm{~nm}$, the coupling to the ferromagnet $A=15 \mu \mathrm{eV}$, and the qubit splitting close to resonance $\left(\Delta_{F}-\Delta=\right.$ $0.5 \mu \mathrm{eV}$, corresponding to about $B=10 \mathrm{mT}$ ), the qubitqubit coupling becomes of the order of $1 \mu \mathrm{eV}$. Such a coupling strength in turn leads to an error threshold on the order of $10^{-8}$. Therefore, even the standard errorcorrection protocol can be used in this case.

\section{Derivation of the effective Hamiltonian (exchange coupling)}

Here, we give a detailed derivation of the qubit-qubit effective Hamiltonian. As stated above, the total Hamiltonian of the system reads

$$
H=H_{F}+H_{\sigma}+A \sum_{i}\left(\frac{1}{2}\left(\sigma_{i}^{+} S_{\mathbf{r}_{i}}^{-}+\sigma_{i}^{-} S_{\mathbf{r}_{i}}^{+}\right)+\sigma_{i}^{z} S_{\mathbf{r}_{i}}^{z}\right),
$$

where we identify the main part as $H_{0}=H_{F}+H_{\sigma}$ and the small perturbation as the exchange coupling $V=$ $A \sum_{i} \boldsymbol{\sigma}_{i} \cdot S_{\mathbf{r}_{i}}$. The Hamiltonian of the ferromagnet reads $H_{F}=-J \sum_{\left\langle\mathbf{r}, \mathbf{r}^{\prime}\right\rangle} \boldsymbol{S}_{\mathbf{r}} \cdot \boldsymbol{S}_{\mathbf{r}^{\prime}}$, while the Hamiltonian for the two distant qubits is $H_{\sigma}=\frac{\Delta}{2} \sum_{i=1,2} \sigma_{i}^{x}$.

The second-order effective Hamiltonian [28] is given by $H_{\text {eff }}^{(2)}=H_{0}+U$, where

$$
U=-\frac{i}{2} \lim _{\eta \rightarrow 0^{+}} \int_{0}^{\infty} d t e^{-\eta t}[V(t), V],
$$

where $V(t)=e^{i H_{0} t} V e^{-i H_{0} t}$.

We have

$\sigma_{i}^{+}(t)=\frac{1+\cos (\Delta t)}{2} \sigma_{i}^{+}+\frac{1-\cos (\Delta t)}{2} \sigma_{i}^{-}-i \sin (\Delta t) \sigma_{i}^{z}$

and $\sigma_{i}^{-}(t)=\sigma_{i}^{+}(t)^{\dagger}$.

Recalling that the longitudinal susceptibility can be neglected and that only the transverse susceptibility contributes, we obtain the following result from Eq. (D7) $U=\lim _{\eta \rightarrow 0^{+}} \int_{0}^{\infty} d t e^{-\eta t} \sum_{i j} U_{i j}$ :

$$
\begin{aligned}
U_{i j} & =-\frac{i A^{2}}{8}\left(\left[\sigma_{i}^{-}(t) S_{\mathbf{r}_{i}}^{+}(t), \sigma_{j}^{+} S_{\mathbf{r}_{j}}^{-}\right]+\text {H.c. }\right) \\
& =-\frac{i A^{2}}{8}\left[\sigma_{i}^{-}(t) \sigma_{j}^{+}\left[S_{\mathbf{r}_{i}}^{+}(t), S_{\mathbf{r}_{j}}^{-}\right]+\text {H.c. }\right] .
\end{aligned}
$$

Finally, by rewriting $\cos (\Delta t)=\frac{e^{i \Delta t}+e^{-i \Delta t}}{2}$ and $\sin (\Delta t)=$ $\frac{e^{i \Delta t}-e^{-i \Delta t}}{2 i}$, and using the definition of the real-space transverse spin susceptibility

$\chi_{\perp}\left(\omega, \mathbf{r}_{i}-\mathbf{r}_{j}\right)=-i \lim _{\eta \rightarrow 0^{+}} \int_{0}^{\infty} d t e^{-(i \omega+\eta) t}\left[S_{\mathbf{r}_{i}}^{+}(t), S_{\mathbf{r}_{j}}^{-}\right]$

we obtain by inserting Eq. (D8) into Eq. (D9)

$$
\begin{aligned}
U= & \frac{A^{2}}{8} \sum_{i j}\left(\frac{\chi_{\perp}(0)}{2}+\frac{\chi_{\perp}(\Delta)+\chi_{\perp}(-\Delta)}{4}\right) \sigma_{i}^{-} \sigma_{j}^{+} \\
& +\frac{A^{2}}{8} \sum_{i j}\left(\frac{\chi_{\perp}(0)}{2}-\frac{\chi_{\perp}(\Delta)+\chi_{\perp}(-\Delta)}{4}\right) \sigma_{i}^{+} \sigma_{j}^{+} \\
& -\frac{A^{2}}{8} \sum_{i j} \frac{\chi_{\perp}(\Delta)-\chi_{\perp}(-\Delta)}{2} \sigma_{i}^{z} \sigma_{j}^{+}+\text {H.c. }
\end{aligned}
$$

Since the decay length of the susceptibility $\chi(\omega, \mathbf{r})$ is large only close to the resonance $\Delta_{F} \sim \Delta$, we can simplify the above equation by neglecting $\chi(-\Delta, \mathbf{r})$ and $\chi(0, \mathbf{r})$ in comparison to $\chi(\Delta, \mathbf{r})$, which is assumed to be close to the resonance. Within this approximation, we arrive at Eq. (D3). 


\section{APPENDIX E: FOURTH-ORDER CONTRIBUTIONS} TO DECOHERENCE

In this section, we determine the effect of the transverse noise in the lowest nonvanishing order due to coupling dipolarly to the ferromagnet. Here, we choose quantization axes such that the qubit splitting is along the $z$ axis, while the ferromagnet is polarized along the $x$ axis. The Hamiltonian of the coupled system reads

$$
H=H_{F}+\frac{\Delta}{2} \sigma^{z}+\sigma^{z} \otimes X+\sigma^{+} \otimes Y^{-}+\sigma^{-} \otimes Y^{+},
$$

where the operator $X(Y)$ that couples longitudinally (transversally) to the qubit is linear in the transverse operators of the ferromagnet

$$
\begin{gathered}
X=\frac{i M_{s}}{2 S} \int d \mathbf{r} c_{\mathbf{r}}\left(S_{\mathbf{r}}^{+}-S_{\mathbf{r}}^{-}\right), \\
Y^{+}=-\frac{i M_{s}}{8 S} \int d \mathbf{r}\left(a_{\mathbf{r}} S_{\mathbf{r}}^{+}+b_{\mathbf{r}} S_{\mathbf{r}}^{-}\right),
\end{gathered}
$$

with $S_{\mathbf{r}}^{ \pm}=S_{\mathbf{r}}^{y} \pm i S_{\mathbf{r}}^{z}$ and the definitions of the coefficients

$$
\begin{gathered}
a_{\mathbf{r}}=B_{\mathbf{r}}+3 C_{\mathbf{r}}-6 A_{\mathbf{r}}, \\
b_{\mathbf{r}}=B_{\mathbf{r}}+3 C_{\mathbf{r}}+6 A_{\mathbf{r}}, \\
c_{\mathbf{r}}=B_{\mathbf{r}}-3 A_{\mathbf{r}}^{\prime \prime}, \\
A_{\mathbf{r}}=\frac{r^{z} r^{+}}{r^{5}}, \\
C_{\mathbf{r}}=\frac{\left(r^{+}\right)^{2}}{r^{5}}, \\
B_{\mathbf{r}}=\frac{1}{r^{3}}\left(2-\frac{3 r^{+} r^{-}}{r^{2}}\right) .
\end{gathered}
$$

To proceed further, we perform the SW transformation on the Hamiltonian given by Eq. (E1). We ignore the Lamb and Stark shifts and obtain the effective Hamiltonian

$H=H_{F}+\frac{\Delta}{2} \sigma^{z}+\sigma^{z} \otimes \tilde{X}_{2}+\sigma^{+} \otimes \tilde{Y}_{2}^{-}+\sigma^{-} \otimes \tilde{Y}_{2}^{+}$,

where

$$
\begin{gathered}
\tilde{X}_{2}=X_{2}-\left\langle X_{2}\right\rangle, \\
\tilde{Y}_{2}^{ \pm}=Y_{2}^{ \pm}-\left\langle Y_{2}^{ \pm}\right\rangle,
\end{gathered}
$$

with the following notation:

$$
\begin{gathered}
X_{2}=4\left(Y_{\Delta}^{+} Y^{-}+Y^{+} Y_{\Delta}^{-}\right), \\
Y_{2}^{+}=2\left(Y_{\Delta}^{+} X-X_{0} Y^{+}\right),
\end{gathered}
$$

$$
X_{\omega}=\frac{i M_{s}}{2 S} \int d \mathbf{r} d \mathbf{r}^{\prime} \chi_{\perp}\left(\omega, \mathbf{r}-\mathbf{r}^{\prime}\right) c_{\mathbf{r}}\left(S_{\mathbf{r}^{\prime}}^{+}-S_{\mathbf{r}^{\prime}}^{-}\right),
$$

$Y_{\omega}^{+}=-\frac{i M_{s}}{8 S} \int d \mathbf{r} d \mathbf{r}^{\prime} \chi_{\perp}\left(\omega, \mathbf{r}-\mathbf{r}^{\prime}\right)\left(a_{\mathbf{r}^{\prime}} S_{\mathbf{r}^{\prime}}^{+}+b_{\mathbf{r}} S_{\mathbf{r}^{\prime}}^{-}\right)$.

The model given by Eq. (E10) yields the following expressions for the relaxation and decoherence times:

$$
\begin{gathered}
T_{1}^{-1}=S_{\tilde{Y}_{2}^{-}}(\omega=\Delta), \\
T_{2}^{-1}=\frac{1}{2} T_{1}^{-1}+S_{\tilde{X}_{2}}(\omega=0),
\end{gathered}
$$

where, again, $S_{A}(\omega)=\int d t e^{-i \omega t}\left\{A^{\dagger}(t), A(0)\right\}$.

After a lengthy calculation, we obtain the expressions for $S_{\tilde{X}_{2}}(\omega=0)$ and $S_{\tilde{Y}_{2}^{-}}(\omega=\Delta)$ :

$$
\begin{aligned}
S_{\tilde{X}_{2}}(0)= & \frac{M_{s}^{4}}{128 S^{4}} \int d \nu d \mathbf{r}_{1} d \mathbf{r}_{2} d \mathbf{r}_{3} d \mathbf{r}_{4} d \mathbf{r}_{5} d \mathbf{r}_{6} C^{-+}\left(\nu, \mathbf{r}_{3}-\mathbf{r}_{4}\right) C^{+-}\left(-\nu, \mathbf{r}_{1}-\mathbf{r}_{2}\right) \times\left[\left(a_{\mathbf{r}_{5}} a_{\mathbf{r}_{3}}^{*}+b_{\mathbf{r}_{3}} b_{\mathbf{r}_{1}}^{*}\right)\left(a_{\mathbf{r}_{4}} a_{\mathbf{r}_{2}}^{*}+b_{\mathbf{r}_{6}} b_{\mathbf{r}_{4}}^{*}\right) \chi_{\perp}\right. \\
& \times\left(\Delta, \mathbf{r}_{1}-\mathbf{r}_{5}\right) \chi_{\perp}\left(\Delta, \mathbf{r}_{2}-\mathbf{r}_{6}\right)+\left(a_{\mathbf{r}_{4}} a_{\mathbf{r}_{2}}^{*}+b_{\mathbf{r}_{5}} b_{\mathbf{r}_{4}}^{*}\right)\left(a_{\mathbf{r}_{1}} a_{\mathbf{r}_{3}}^{*}+b_{\mathbf{r}_{6}} b_{\mathbf{r}_{1}}^{*}\right) \chi_{\perp}\left(\Delta, \mathbf{r}_{2}-\mathbf{r}_{5}\right) \chi_{\perp}\left(\Delta, \mathbf{r}_{3}-\mathbf{r}_{6}\right) \\
& +\left(a_{\mathbf{r}_{6}} a_{\mathbf{r}_{2}}^{*}+b_{\mathbf{r}_{2}} b_{\mathbf{r}_{4}}^{*}\right)\left(a_{\mathbf{r}_{5}} a_{\mathbf{r}_{3}}^{*}+b_{\mathbf{r}_{3}} b_{\mathbf{r}_{1}}^{*}\right) \chi_{\perp}\left(\Delta, \mathbf{r}_{1}-\mathbf{r}_{5}\right) \chi_{\perp}\left(\Delta, \mathbf{r}_{4}-\mathbf{r}_{6}\right) \\
& \left.+\left(a_{\mathbf{r}_{6}} a_{\mathbf{r}_{2}}^{*}+b_{\mathbf{r}_{2}} b_{\mathbf{r}_{4}}^{*}\right)\left(a_{\mathbf{r}_{1}} a_{\mathbf{r}_{3}}^{*}+b_{\mathbf{r}_{5}} b_{\mathbf{r}_{1}}^{*}\right) \chi_{\perp}\left(\Delta, \mathbf{r}_{3}-\mathbf{r}_{5}\right) \chi_{\perp}\left(\Delta, \mathbf{r}_{4}-\mathbf{r}_{6}\right)\right]
\end{aligned}
$$




$$
\begin{aligned}
& S_{\tilde{Y}_{2}^{-}}(\Delta)=\frac{M_{s}^{4}}{64 S^{4}} \int d \nu d \mathbf{r}_{1} d \mathbf{r}_{2} d \mathbf{r}_{3} d \mathbf{r}_{4} d \mathbf{r}_{5} d \mathbf{r}_{6} C^{-+}\left(\nu, \mathbf{r}_{3}-\mathbf{r}_{4}\right) C^{+-}\left(\Delta-\nu, \mathbf{r}_{1}-\mathbf{r}_{2}\right) \\
& \times\left[c_{\mathbf{r}_{3}} c_{\mathbf{r}_{6}}\left(a_{\mathbf{r}_{4}} b_{\mathbf{r}_{1}}^{*}+a_{\mathbf{r}_{5}} b_{\mathbf{r}_{4}}^{*}\right) \chi_{\perp}\left(0, \mathbf{r}_{2}-\mathbf{r}_{6}\right) \chi_{\perp}\left(\Delta, \mathbf{r}_{1}-\mathbf{r}_{5}\right)-c_{\mathbf{r}_{3}} c_{\mathbf{r}_{6}}\left(a_{\mathbf{r}_{5}} a_{\mathbf{r}_{2}}^{*}+b_{\mathbf{r}_{2}} b_{\mathbf{r}_{1}}^{*}\right) \chi_{\perp}\left(0, \mathbf{r}_{4}-\mathbf{r}_{6}\right) \chi_{\perp}\left(\Delta, \mathbf{r}_{1}-\mathbf{r}_{5}\right)\right. \\
& -c_{\mathbf{r}_{4}} c_{\mathbf{r}_{6}}\left(a_{\mathbf{r}_{1}} a_{\mathbf{r}_{2}}^{*}+b_{\mathbf{r}_{5}} b_{\mathbf{r}_{1}}^{*}\right) \chi_{\perp}\left(0, \mathbf{r}_{3}-\mathbf{r}_{6}\right) \chi_{\perp}\left(\Delta, \mathbf{r}_{2}-\mathbf{r}_{5}\right)+c_{\mathbf{r}_{1}} c_{\mathbf{r}_{6}}\left(b_{\mathbf{r}_{5}} a_{\mathbf{r}_{2}}^{*}+b_{\mathbf{r}_{2}} a_{\mathbf{r}_{3}}^{*}\right) \chi_{\perp}\left(0, \mathbf{r}_{4}-\mathbf{r}_{6}\right) \chi_{\perp}\left(\Delta, \mathbf{r}_{3}-\mathbf{r}_{5}\right) \\
& +c_{\mathbf{r}_{4}} c_{\mathbf{r}_{5}}\left(b_{\mathbf{r}_{3}} a_{\mathbf{r}_{2}}^{*}+b_{\mathbf{r}_{6}} a_{\mathbf{r}_{3}}^{*}\right) \chi_{\perp}\left(0, \mathbf{r}_{1}-\mathbf{r}_{5}\right) \chi_{\perp}\left(\Delta, \mathbf{r}_{2}-\mathbf{r}_{6}\right)+c_{\mathbf{r}_{3}} c_{\mathbf{r}_{4}}\left(a_{\mathbf{r}_{5}} a_{\mathbf{r}_{2}}^{*}+b_{\mathbf{r}_{6}} b_{\mathbf{r}_{1}}^{*}\right) \chi_{\perp}\left(\Delta, \mathbf{r}_{1}-\mathbf{r}_{5}\right) \chi_{\perp}\left(\Delta, \mathbf{r}_{2}-\mathbf{r}_{6}\right) \\
& -c_{\mathbf{r}_{1}} c_{\mathbf{r}_{5}}\left(a_{\mathbf{r}_{4}} a_{\mathbf{r}_{3}}^{*}+b_{\mathbf{r}_{6}} b_{\mathbf{r}_{4}}^{*}\right) \chi_{\perp}\left(0, \mathbf{r}_{2}-\mathbf{r}_{5}\right) \chi_{\perp}\left(\Delta, \mathbf{r}_{3}-\mathbf{r}_{6}\right)-c_{\mathbf{r}_{1}} c_{\mathbf{r}_{4}}\left(b_{\mathbf{r}_{6}} a_{\mathbf{r}_{2}}^{*}+b_{\mathbf{r}_{5}} a_{\mathbf{r}_{3}}^{*}\right) \chi_{\perp}\left(\Delta, \mathbf{r}_{2}-\mathbf{r}_{5}\right) \chi_{\perp}\left(\Delta, \mathbf{r}_{3}-\mathbf{r}_{6}\right) \\
& -c_{\mathbf{r}_{2}} c_{\mathbf{r}_{5}}\left(a_{\mathbf{r}_{6}} a_{\mathbf{r}_{3}}^{*}+b_{\mathbf{r}_{3}} b_{\mathbf{r}_{4}}^{*}\right) \chi_{\perp}\left(0, \mathbf{r}_{1}-\mathbf{r}_{5}\right) \chi_{\perp}\left(\Delta, \mathbf{r}_{4}-\mathbf{r}_{6}\right)+c_{\mathbf{r}_{2}} c_{\mathbf{r}_{5}}\left(a_{\mathbf{r}_{6}} b_{\mathbf{r}_{1}}^{*}+a_{\mathbf{r}_{1}} b_{\mathbf{r}_{4}}^{*}\right) \chi_{\perp}\left(0, \mathbf{r}_{3}-\mathbf{r}_{5}\right) \chi_{\perp}\left(\Delta, \mathbf{r}_{4}-\mathbf{r}_{6}\right) \\
& -c_{\mathbf{r}_{2}} c_{\mathbf{r}_{3}}\left(a_{\mathbf{r}_{6}} b_{\mathbf{r}_{1}}^{*}+a_{\mathbf{r}_{5}} b_{\mathbf{r}_{4}}^{*}\right) \chi_{\perp}\left(\Delta, \mathbf{r}_{1}-\mathbf{r}_{5}\right) \chi_{\perp}\left(\Delta, \mathbf{r}_{4}-\mathbf{r}_{6}\right)+c_{\mathbf{r}_{1}} c_{\mathbf{r}_{2}}\left(a_{\mathbf{r}_{6}} a_{\mathbf{r}_{3}}^{*}+b_{\mathbf{r}_{5}} b_{\mathbf{r}_{4}}^{*}\right) \chi_{\perp}\left(\Delta, \mathbf{r}_{3}-\mathbf{r}_{5}\right) \chi_{\perp}\left(\Delta, \mathbf{r}_{4}-\mathbf{r}_{6}\right) \\
& +c_{\mathbf{r}_{5}} c_{\mathbf{r}_{6}}\left(a_{\mathbf{r}_{4}} a_{\mathbf{r}_{3}}^{*}+b_{\mathbf{r}_{3}} b_{\mathbf{r}_{4}}^{*}\right) \chi_{\perp}\left(0, \mathbf{r}_{1}-\mathbf{r}_{5}\right) \chi_{\perp}\left(0, \mathbf{r}_{2}-\mathbf{r}_{6}\right)-c_{\mathbf{r}_{5}} c_{\mathbf{r}_{6}}\left(a_{\mathbf{r}_{4}} b_{\mathbf{r}_{1}}^{*}+a_{\mathbf{r}_{1}} b_{\mathbf{r}_{4}}^{*}\right) \chi_{\perp}\left(0, \mathbf{r}_{2}-\mathbf{r}_{5}\right) \chi_{\perp}\left(0, \mathbf{r}_{3}-\mathbf{r}_{6}\right) \\
& \left.-c_{\mathbf{r}_{5}} c_{\mathbf{r}_{6}}\left(b_{\mathbf{r}_{3}} a_{\mathbf{r}_{2}}^{*}+b_{\mathbf{r}_{2}} a_{\mathbf{r}_{3}}^{*}\right) \chi_{\perp}\left(0, \mathbf{r}_{1}-\mathbf{r}_{5}\right) \chi_{\perp}\left(0, \mathbf{r}_{4}-\mathbf{r}_{6}\right)+c_{\mathbf{r}_{5}} c_{\mathbf{r}_{6}}\left(a_{\mathbf{r}_{1}} a_{\mathbf{r}_{2}}^{*}+b_{\mathbf{r}_{2}} b_{\mathbf{r}_{1}}^{*}\right) \chi_{\perp}\left(0, \mathbf{r}_{3}-\mathbf{r}_{5}\right) \chi_{\perp}\left(0, \mathbf{r}_{4}-\mathbf{r}_{6}\right)\right] .
\end{aligned}
$$

In order to obtain the lower bound of the relaxation and decoherence times, we consider the ferromagnet to be in the shape of an infinite plane. Furthermore, we are not aiming at performing an exact evaluation of the integrals in Eqs. (E19) and (E20) but rather at finding the lower bound for the relaxation and decoherence times. To this end, we note that $\left|C^{+-}\left(\omega, \mathbf{r}-\mathbf{r}^{\prime}\right)\right| \leq\left|C^{+-}(\omega, \mathbf{r}=0)\right|$ and arrive at the following inequalities:

$$
\begin{aligned}
S_{\tilde{X}_{2}}(0) \leq & \frac{B^{4} M_{s}^{4}}{8 S^{2}\left(\Delta_{F}-\Delta\right)^{2}} \int_{\Delta_{F}}^{\infty} d \nu C^{+-}(\nu)^{2}, \\
S_{\tilde{Y}_{2}^{-}}(\Delta) \leq & \frac{B^{4} M_{s}^{4}}{8 S^{2}}\left(\frac{1}{\Delta_{F}}+\frac{1}{\Delta_{F}-\Delta}\right)^{2} \\
& \times \int_{\Delta_{F}+\Delta}^{\infty} d \nu C^{+-}(\nu) C^{+-}(\nu-\Delta),
\end{aligned}
$$

where we use the notation $B=\int d \mathbf{r} B_{\mathbf{r}}$. Finally, we arrive at the expression for the lower bound of the relaxation and decoherence times

$$
\begin{aligned}
T_{1}^{-1} \leq & \frac{B^{4} M_{s}^{4} \Delta_{F}^{2}}{2 D^{3} S^{2}}\left(\frac{1}{\Delta_{F}}+\frac{1}{\Delta_{F}-\Delta}\right)^{2} f\left(\frac{\Delta}{\Delta_{F}}, \beta \Delta_{F}\right), \\
T_{2}^{-1} \leq & \frac{B^{4} M_{s}^{4} \Delta_{F}^{2}}{4 D^{3} S^{2}}\left(\frac{1}{\Delta_{F}}+\frac{1}{\Delta_{F}-\Delta}\right)^{2} f\left(\frac{\Delta}{\Delta_{F}}, \beta \Delta_{F}\right) \\
& +\frac{B^{4} M_{s}^{4} \Delta_{F}^{2}}{2 D^{3} S^{2}\left(\Delta_{F}-\Delta\right)^{2}} f\left(0, \beta \Delta_{F}\right),
\end{aligned}
$$

with the function $f(x, y)$ defined as follows:

$$
f(x, y)=\int_{1+x}^{\infty} d z \frac{\sqrt{z-1}}{e^{y z}-1} \frac{\sqrt{z-x-1}}{e^{y(z-x)}-1} .
$$

Assuming the same parameters as in the main text, we obtain decoherence times of about $0.5 \mathrm{~h}$, while the relaxation time is on the order of $1000 \mathrm{~h}$. It is worth noting that this result depends sensitively on the ratio $\Delta_{F} / T$; thus, if we assume a temperature of $4 \mathrm{~K}$, we obtain $T_{1} \geq 200 \mu \mathrm{s}$ and $T_{2} \geq 30 \mu \mathrm{s}$.
[1] F. Jelezko, T. Gaebel, I. Popa, A. Gruber, and J. Wrachtrup, Observation of Coherent Oscillations in a Single Electron Spin, Phys. Rev. Lett. 92, 076401 (2004).

[2] F. Jelezko, T. Gaebel, I. Popa, M. Domhan, A. Gruber, and J. Wrachtrup, Observation of Coherent Oscillation of a Single Nuclear Spin and Realization of a Two-Qubit Conditional Quantum Gate, Phys. Rev. Lett. 93, 130501 (2004).

[3] L. Childress, M.V. Gurudev Dutt, J.M. Taylor, A. S. Zibrov, F. Jelezko, J. Wrachtrup, P. R. Hemmer, and M. D. Lukin, Coherent Dynamics of Coupled Electron and Nuclear Spin Qubits in Diamond, Science 314, 281 (2006).

[4] M. V. Gurudev Dutt, L. Childress, L. Jiang, E. Togan, J. Maze, F. Jelezko, A. S. Zibrov, P. R. Hemmer, and M. D. Lukin, Quantum Register Based on Individual Electronic and Nuclear Spin Qubits in Diamond, Science 316, 1312 (2007).

[5] R. Hanson, V. V. Dobrovitski, A. E. Feiguin, O. Gywat, and D. D. Awschalom, Coherent Dynamics of a Single Spin Interacting with an Adjustable Spin Bath, Science 320, 352 (2008).

[6] P. Neumann, N. Mizuochi, F. Rempp, P. Hemmer, H. Watanabe, S. Yamasaki, V. Jacques, T. Gaebel, F. Jelezko, and J. Wrachtrup, Multipartite Entanglement among Single Spins in Diamond, Science 320, 1326 (2008).

[7] L. Jiang, J. S. Hodges, J. R. Maze, P. Maurer, J. M. Taylor, D. G. Cory, P. R. Hemmer, R. L. Walsworth, A. Yacoby, A. S. Zibrov, and M.D. Lukin, Repetitive Readout of a Single Electronic Spin via Quantum Logic with Nuclear Spin Ancillae, Science 326, 267 (2009).

[8] G. D. Fuchs, V. V. Dobrovitski, D. M. Toyli, F. J. Heremans, and D. D. Awschalom, Gigahertz Dynamics of a Strongly Driven Single Quantum Spin, Science 326, 1520 (2009).

[9] P. Neumann, J. Beck, M. Steiner, F. Rempp, H. Fedder, P. R. Hemmer, J. Wrachtrup, and F. Jelezko, Single-Shot Readout of a Single Nuclear Spin, Science 329, 542 (2010).

[10] B. B. Buckley, G.D. Fuchs, L.C. Bassett, and D. D. Awschalom, Spin-Light Coherence for Single-Spin 
Measurement and Control in Diamond, Science 330, 1212 (2010).

[11] E. Togan, Y. Chu, A. S. Trifonov, L. Jiang, J. Maze, L. Childress, M. V. G. Dutt, A. S. Sorensen, P. R. Hemmer, A. S. Zibrov, and M.D. Lukin, Quantum Entanglement between an Optical Photon and a Solid-State Spin Qubit, Nature (London) 466, 730 (2010).

[12] L. Robledo, L. Childress, H. Bernien, B. Hensen, P. F. A. Alkemade, and R. Hanson, High-Fidelity Projective Readout of a Solid-State Spin Quantum Register, Nature (London) 477, 574 (2011).

[13] J. J. Pla, K. Y. Tan, J. P. Dehollain, W. H. Lim, J. J. L. Morton, F. A. Zwanenburg, D. N. Jamieson, A.S. Dzurak, and A. Morello, High-Fidelity Readout and Control of a Nuclear Spin Qubit in Silicon, Nature (London) 496, 334 (2013).

[14] J. J. Pla, K. Y. Tan, J. P. Dehollain, W. H. Lim, J. J. L. Morton, D. N. Jamieson, A. S. Dzurak, and A. Morello, A Single-Atom Electron Spin Qubit in Silicon, Nature (London) 489, 541 (2012).

[15] V. V. Dobrovitski, G. D. Fuchs, A. L. Falk, C. Santori, and D. D. Awschalom, Quantum Control over Single Spins in Diamond, Annu. Rev. Condens. Matter Phys. 4, 23 (2013).

[16] G. Balasubramanian, P. Neumann, D. Twitchen, M. Markham, R. Kolesov, N. Mizuochi, J. Isoya, J. Achard, J. Beck, J. Tissler, V. Jacques, P. R. Hemmer, F. Jelezko, and J. Wrachtrup, Ultralong Spin Coherence Time in Isotopically Engineered Diamond, Nat. Mater. 8, 383 (2009).

[17] A. Sipahigil, M. L. Goldman, E. Togan, Y. Chu, M. Markham, D. J. Twitchen, A. S. Zibrov, A. Kubanek, and M.D. Lukin, Quantum Interference of Single Photons from Remote Nitrogen-Vacancy Centers in Diamond, Phys. Rev. Lett. 108, 143601 (2012).

[18] T. van der Sar, Z. H. Wang, M. S. Blok, H. Bernien, T. H. Taminiau, D. M. Toyli, D. A. Lidar, D. D. Awschalom, R. Hanson, and V. V. Dobrovitski, Decoherence-Protected Quantum Gates for a Hybrid Solid-State Spin Register, Nature (London) 484, 82 (2012).

[19] B. Lenk, H. Ulrichs, F. Garbs, and M. Münzenberg, The Building Blocks of Magnonics, Phys. Rep. 507, 107 (2011).

[20] L. Trifunovic, F. L. Pedrocchi, and D. Loss, Long-Range Interaction of Singlet-Triplet Qubits via Ferromagnets, arXiv: 1305.2451.

[21] D. Loss and D. P. DiVincenzo, Quantum Computation with Quantum Dots, Phys. Rev. A 57, 120 (1998).

[22] C. Kloeffel and D. Loss, Prospects for Spin-Based Quantum Computing in Quantum Dots, Annu. Rev. Condens. Matter Phys. 4, 51 (2013).

[23] L. Trifunovic, O. Dial, M. Trif, J. R. Wootton, R. Abebe, A. Yacoby, and D. Loss, Long-Distance Spin-Spin Coupling via Floating Gates, Phys. Rev. X 2, 011006 (2012).

[24] M. D. Shulman, O. E. Dial, S. P. Harvey, H. Bluhm, V. Umansky, and A. Yacoby, Demonstration of Entanglement of Electrostatically Coupled Singlet-Triplet Qubits, Science 336, 202 (2012).

[25] L. Childress, A. S. Sørensen, and M. D. Lukin, Mesoscopic Cavity Quantum Electrodynamics with Quantum Dots, Phys. Rev. A 69, 042302 (2004).
[26] G. Burkard and A. Imamoglu, Ultra-long-Distance Interaction between Spin Qubits, Phys. Rev. B 74, 041307(R) (2006).

[27] M. Trif, V. N. Golovach, and D. Loss, Spin Dynamics in InAs Nanowire Quantum Dots Coupled to a Transmission Line, Phys. Rev. B 77, 045434 (2008).

[28] S. Bravyi, D. P. DiVincenzo, and D. Loss, Schrieffer-Wolff Transformation for Quantum Many-Body Systems, Ann. Phys. (Amsterdam) 326, 2793 (2011).

[29] C. Kittel, On the Theory of Ferromagnetic Resonance Absorption, Phys. Rev. 73, 155 (1948).

[30] H. Watanabe and H. Murayama, Unified Description of Nambu-Goldstone Bosons without Lorentz Invariance, Phys. Rev. Lett. 108, 251602 (2012).

[31] M. Sparks, Ferromagnetic Resonance in Thin Films. I. Theory of Normal-Mode Frequencies, Phys. Rev. B 1, 3831 (1970).

[32] I. Lee, Y. Obukhov, G. Xiang, A. Hauser, F. Yang, P. Banerjee, D. V. Pelekhov, and P. C. Hammel, Nanoscale Scanning Probe Ferromagnetic Resonance Imaging Using Localized Modes, Nature (London) 466, 845 (2010).

[33] M. A. Nielsen and I. L. Chuang, Quantum Computation and Quantum Information (Cambridge University Press, Cambridge, England, 2004), 1st ed.

[34] A. Imamoglu, D. D. Awschalom, G. Burkard, D. P. DiVincenzo, D. Loss, M. Sherwin, and A. Small, Quantum Information Processing Using Quantum Dot Spins and Cavity QED, Phys. Rev. Lett. 83, 4204 (1999).

[35] T. Tanamoto, K. Maruyama, Y.-x. Liu, X. Hu, and F. Nori, Efficient Purification Protocols Using iSWAP Gates in Solid-State Qubits, Phys. Rev. A 78, 062313 (2008).

[36] D.S. Wang, A. G. Fowler, and L.C.L. Hollenberg, Surface Code Quantum Computing with Error Rates over 1\%, Phys. Rev. A 83, 020302(R) (2011).

[37] D.P. DiVincenzo and D. Loss, Rigorous Born Approximation and Beyond for the Spin-Boson Model, Phys. Rev. B 71, 035318 (2005).

[38] Y. Makhlin and A. Shnirman, Dephasing of Solid-State Qubits at Optimal Points, Phys. Rev. Lett. 92, 178301 (2004).

[39] A. A. Abrikosov, L.P. Gor'kov, and I. E. Dzyaloshinski, Methods of Quantum Field Theory in Statistical Physics (Dover, New York, 1975).

[40] Y. Makhlin, G. Schön, and A. Shnirman, Dissipative Effects in Josephson Qubits, Chem. Phys. 296, 315 (2004).

[41] J.-P. Tetienne, L. Rondin, P. Spinicelli, M. Chipaux, T. Debuisschert, J.-F. Roch, and V. Jacques, Magnetic-FieldDependent Photodynamics of Single NV Defects in Diamond: An Application to Qualitative All-Optical Magnetic Imaging, New J. Phys. 14, 103033 (2012).

[42] W. Nolting and A. Ramakanth, Quantum Theory of Magnetism (Springer, New York, 2009).

[43] J. R. Hauptmann, J. Paaske, and P.E. Lindelof, ElectricField-Controlled Spin Reversal in a Quantum Dot with Ferromagnetic Contacts, Nat. Phys. 4, 373 (2008).

[44] L. Hofstetter, A. Geresdi, M. Aagesen, J. Nygård, C. Schönenberger, and S. Csonka, Ferromagnetic Proximity Effect in a Ferromagnet-Quantum-Dot-Superconductor Device, Phys. Rev. Lett. 104, 246804 (2010).

[45] S. Amasha, K. MacLean, Iuliana P. Radu, D. M. Zumbühl, M. A. Kastner, M.P. Hanson, and A.C. Gossard, 
Electrical Control of Spin Relaxation in a Quantum Dot, Phys. Rev. Lett. 100, 046803 (2008).

[46] H. Bluhm, S. Foletti, I. Neder, M. Rudner, D. Mahalu, V. Umansky, and A. Yacoby, Dephasing Time of GaAs Electron-Spin Qubits Coupled to a Nuclear Bath Exceeding $200 \mu \mathrm{s}$, Nat. Phys. 7, 109 (2010).

[47] R. Raussendorf and J. Harrington, Fault-Tolerant Quantum Computation with High Threshold in Two Dimensions, Phys. Rev. Lett. 98, 190504 (2007).

[48] K. Khodjasteh and L. Viola, Dynamically Error-Corrected Gates for Universal Quantum Computation, Phys. Rev. Lett. 102, 080501 (2009).
[49] X. Xu, W. Yao, B. Sun, D. G. Steel, A. S. Bracker, D. Gammon, and L. J. Sham, Optically Controlled Locking of the Nuclear Field via Coherent Dark-State Spectroscopy, Nature (London) 459, 1105 (2009).

[50] I. T. Vink, K. C. Nowack, F. H. L. Koppens, J. Danon, Y. V. Nazarov, and L.M.K. Vandersypen, Locking Electron Spins into Magnetic Resonance by Electron-Nuclear Feedback, Nat. Phys. 5, 764 (2009).

[51] H. Ohno, D. Chiba, F. Matsukura, T. Omiya, E. Abe, T. Dietl, Y. Ohno, and K. Ohtani, Electric-Field Control of Ferromagnetism, Nature (London) 408, 944 (2000). 